\title{
A COMPACT CONCENTRATION OF LARGE GRAINS IN THE HD 142527 PROTOPLANETARY DUST TRAP
}

\author{
Simon Casassus ${ }^{1,2}$, Chris M. Wright ${ }^{3}$, Sebastian Marino ${ }^{1,2}$, Sarah T. Maddison ${ }^{4}$, Al Wootten $^{5}$, Pablo Roman $^{2,6}$, \\ Sebastian Pérez ${ }^{1,2}$, Paola Pinilla ${ }^{7}$, Mark Wyatt ${ }^{8}$, Victor Moral ${ }^{2,6}$, Francois Ménard ${ }^{9}$, Valentin Christiaens ${ }^{1,2}$, \\ Lucas Cieza ${ }^{10}$, AND Gerrit VAN der Plas ${ }^{1,2}$ \\ ${ }^{1}$ Departamento de Astronomía, Universidad de Chile, Casilla 36-D, Santiago, Chile \\ ${ }^{2}$ Millennium Nucleus "Protoplanetary Disks," Santiago, Chile \\ ${ }^{3}$ School of Physical, Environmental and Mathematical Sciences, UNSW Canberra, P.O. Box 7916, Canberra BC 2610, Australia \\ ${ }^{4}$ Centre for Astrophysics \& Supercomputing, Swinburne University of Technology, P.O. Box 218, Hawthorn, VIC 3122, Australia \\ ${ }^{5}$ National Radio Astronomy Observatory, 520 Edgemont Road, Charlottesville, VA 22903-2475, USA \\ ${ }^{6}$ Center for Mathematical Modeling, Universidad de Chile, Av. Blanco Encalada 2120 Piso 7, Santiago, Chile \\ ${ }_{7}$ Leiden Observatory, Leiden University, P.O. Box 9513, 2300RA Leiden, The Netherlands \\ ${ }^{8}$ Institute of Astronomy, University of Cambridge, Madingley Road, Cambridge CB3 0HA, UK \\ ${ }^{9}$ UMI-FCA, CNRS/INSU France (UMI 3386), at Departamento de Astronomía, Universidad de Chile, Santiago, Chile \\ ${ }^{10}$ Facultad de Ingeniería, Universidad Diego Portales, Av. Ejército 441, Santiago, Chile \\ Received 2015 May 28; accepted 2015 September 6; published 2015 October 15
}

\begin{abstract}
A pathway to the formation of planetesimals, and eventually giant planets, may occur in concentrations of dust grains trapped in pressure maxima. Dramatic crescent-shaped dust concentrations have been seen in recent radio images at submillimeter wavelengths. These disk asymmetries could represent the initial phases of planet formation in the dust trap scenario, provided that grain sizes are spatially segregated. A testable prediction of azimuthal dust trapping is that progressively larger grains should be more sharply confined and should follow a distribution that is markedly different from the gas. However, gas tracers such as ${ }^{12} \mathrm{CO}$ and the infrared emission from small grains are both very optically thick where the submillimeter continuum originates, so previous observations have been unable to test the trapping predictions or to identify compact concentrations of larger grains required for planet formation by core accretion. Here we report multifrequency observations of HD 142527, from 34 to $700 \mathrm{GHz}$, that reveal a compact concentration of grains approaching centimeter sizes, with a few Earth masses, embedded in a large-scale crescent of smaller, submillimeter-sized particles. The emission peaks at wavelengths shorter than $\sim 1 \mathrm{~mm}$ are optically thick and trace the temperature structure resulting from shadows cast by the inner regions. Given this temperature structure, we infer that the largest dust grains are concentrated in the $34 \mathrm{GHz}$ clump. We conclude that dust trapping is efficient enough for grains observable at centimeter wavelengths to lead to compact concentrations.
\end{abstract}

Key words: planet-disk interactions - protoplanetary disks - stars: individual (HD 142527)

\section{INTRODUCTION}

Giant planet formation occurs in the first few million years following stellar birth, while the parent protoplanetary disk is still gas-rich (Zuckerman et al. 1995). However, the classical debate on the formation mechanism, if envelope accretion onto a rocky core, that is, core accretion (Pollack et al. 1996), or gravitational instability (Kuiper 1951), is stalled without observations. The pathway to giant planet formation determines the initial configuration and compositions of the product planetary systems. Current trends in theory contemplate a variety of formation scenarios, notably second-generation core accretion at large radii following the formation of a giant closer in (Sándor et al. 2011; Ayliffe et al. 2012). Models predict that young giant protoplanets carve a deep gap in the dust component of protoplanetary disks and a shallower gap in the gas (Paardekooper \& Mellema 2006; Fouchet et al. 2010). The clearing of the protoplanetary gap is thought to underlie the class of "transition" disks. A local pressure maximum develops at the outer edge of the gap, which can trap and pile up the larger grains (Zhu \& Stone 2014) that would otherwise rapidly migrate inward due to aerodynamic drag (Weidenschilling 1977 , as in the so-called "meter-size barrier," which at 50-100 AU corresponds to millimeter-sized particles). Local pressure maxima could also occur in lopsided disks with a stellar offset (Mittal \& Chiang 2015). Whichever is its origin, the development of a local pressure maximum may promote the pile-up and growth of dust grains and the formation of large dust clumps that could eventually collapse into planetary cores (Lyra et al. 2009; Sándor et al. 2011; Ayliffe et al. 2012).

Recent observational progress brought by the Atacama Large Millimeter Array (ALMA) fits in the scenario of secondgeneration planet formation at large stellocentric radii. The observational identification of so-called dust traps, in the form of concentrations of millimeter-sized dust (Casassus et al. 2013; van der Marel et al. 2013) in the outer regions of transition disks, suggests that azimuthal dust trapping may occur. An important prediction of this scenario is that progressively larger grains ${ }^{11}$ should be more sharply confined (Birnstiel et al. 2013; Lyra \& Lin 2013), possibly leading to the formation of boulders and planetesimals. Another consequence is that the larger dust grains should have a distribution that is markedly different from the gas. Observed contrast ratios of $30-100$ between extrema in the submillimeter continuum (at coarse angular resolutions) would seem too large to reflect an equally lopsided gas distribution. However, a question remains as to the impact of enhanced cooling in the dust trap on the density contrast. In observational terms, the use of gas tracers such as ${ }^{12} \mathrm{CO}$ or the infrared (IR) emission from small grains are both very optically thick where the ALMA thermal

\footnotetext{
11 Up to Stokes numbers of $\sim 1$, which for the physical conditions in HD 142527 correspond to grains $\sim 30 \mathrm{~cm}$ in size that are not observable with current instrumentation.
} 


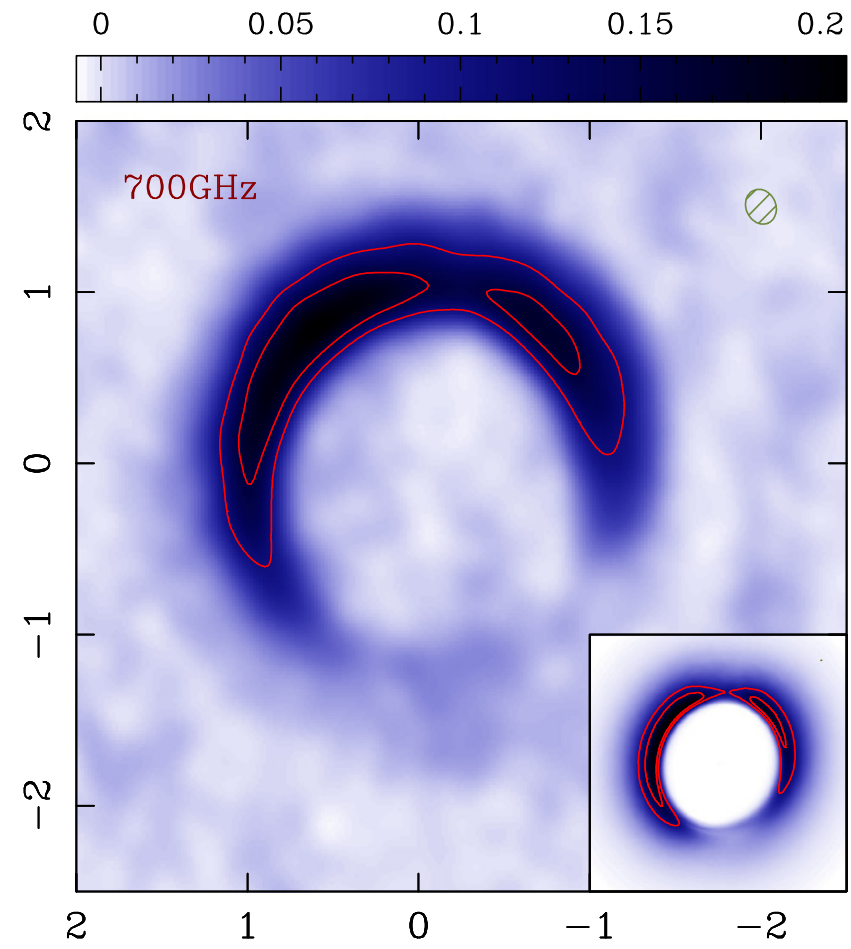

Figure 1. ALMA band 9 observations of HD 142527, and comparison with synthetic predictions. The $x$ and $y$ axes indicate angular offset in arcsec along right ascension (R.A.) and declination (decl.) relative to the stellar position, at the origin of coordinates. The color scale shows a restored image of the ALMA band 9 at $700 \mathrm{GHz}$, with contours at 0.5 and 0.75 times the peak intensity. The wedge indicates specific intensity in $\mathrm{Jy} \mathrm{beam}^{-1}$, with a beam of $0.21 \times 0.17$ arcsec (the beam ellipse is shown on the upper right corner) The inset shows emergent intensities predicted from the dust trap model (see Section 4), including the impact on grain temperatures due to shadowing from a tilted inner disk. Contours are at 0.5 and 0.75 times the peak, and no smoothing has been applied. The side of the inset corresponds to $3.5 \operatorname{arcsec}$.

continuum originates, so the previous observations (Casassus et al. 2013; van der Marel et al. 2013; Pérez et al. 2014) could not test the trapping predictions. For instance, in IRS 48 the outer ring is optically thick in the mid-IR emission (Bruderer et al. 2014, their Section 4.1), as is ${ }^{12} \mathrm{CO}(6-5)$, whose azimuthal structure is also modulated by foreground absorption.

Since emission at a given wavelength is dominated by grains of a matching size, multiwavelength radio observations can confirm whether the crescent-shaped continua seen in transition disks are indeed due to the dust trap phenomenon. In this work we compare new ALMA high-frequency radio continuum observations of HD 142527, obtained at $700 \mathrm{GHz}$ (ALMA band 9; wavelengths of $\sim 0.43 \mathrm{~mm}$, described in Appendix A.1), with resolved images at $34 \mathrm{GHz}(8.8 \mathrm{~mm})$ acquired at the Australia Telescope Compact Array (ATCA, see Appendix A.2). In Section 2 we show that the optical depths are of order $\sim 1$ at $345 \mathrm{GHz}$, so the submillimeter spectral trends observed with ALMA correspond to optical depth effects rather than to dust trapping. In Section 3 we explain that the ATCA observations reveal a compact clump at $34 \mathrm{GHz}$ embedded in the submillimeter crescent, which is reflected by an opacity spectral index corresponding to larger grains, as expected in the dust trapping scenario. In Section 4 we discuss the observational results in terms of radiative transfer predictions based on a parametric dust trap model. Section 5 summarizes our results.

\section{AN OPTICALLY THICK SUBMILLIMETER CONTINUUM}

The ALMA band 9 continuum (Figure 1) has a morphology that is intermediate between the broken ring seen in the thermal IR at $18 \mu \mathrm{m}$ (Fujiwara et al. 2006; Verhoeff et al. 2011) and the $345 \mathrm{GHz}$ crescent seen in ALMA band 7 (Casassus et al. 2013). The maximum radial width of the crescent, measured at halfmaximum, is about 0 .' 6 , so it is resolved with a beam size of 0 ." 25

A traditional means to quantify the spectral variations as a function of position is the spectral index map $\alpha(\boldsymbol{x})$, with a power-law parameterization of the specific intensity $I_{\nu}=I_{\nu \circ}\left(\nu / \nu_{\circ}\right)^{\alpha(x)}$. We find that the spectral index between band 7 and band $9, \alpha_{\mathrm{b} 7}^{\mathrm{b} 9}$, as well as the slope of the spectrum across the $0.13 \mathrm{GHz}$ frequency lever within ALMA band 7, $\alpha_{\mathrm{b} 7}$, both anticorrelate with the continuum crescent (see Figure 2; details are given in Section A.6), meaning that the azimuthal extension is greater at shorter wavelength. However, while the band 7 and band 9 continua peak between 10 and $11 \mathrm{hr}$ as a function of azimuth along the outer ring, the spectral index maps exhibit a common minimum at $\sim 1 \mathrm{hr}$, that is, at the northern ansa of the ring. This coincidence is indicative of a projection effect, as in limb brightening: if column densities within the band 7 beam are greater at $1 \mathrm{hr}$, the increased optical depths will result in flatter spectra.

The spectral variations inferred from the multifrequency morphological trends can be cast into sky maps for the optical depth $\tau(\boldsymbol{x})=\tau_{\circ}(\boldsymbol{x}) \times\left(\nu / \nu_{\circ}\right)^{\beta_{s}(\boldsymbol{x})}$ and line-of-sight temperature $T_{S}(\boldsymbol{x})$, as a function of angular position $\boldsymbol{x}$. We fit the observations with the intensities emergent from a uniform slab (hereafter "graybody," see Appendix A.5):

$$
I_{\nu}^{m}(\boldsymbol{x})=B_{\nu}\left(T_{s}(\boldsymbol{x})\right)[1-\exp (-\tau(\boldsymbol{x}))]
$$

These graybody models are used as diagnostics for the optical depth and are meant to characterize the emergent spectrum at a given line of sight with three parameters adjusted to three frequency points. The inferred temperature field approximates the opacity-weighted average temperature along the line of sight (see Section 4.1 for a discussion of biases).

By applying the graybody diagnostic to the ALMA band 7 amplitude and slope, and including band 9, we find that the crescent is optically thick in band 7. In this case, we have only two independent measurements, so we use a constant emissivity law $\beta_{S}(\boldsymbol{x})=1.5$. Despite the use of constant $\beta_{S}$, Figure 3 shows that graybody line-of-sight models provide a fit to the observations. The crescent reaches optical depths close to 1 at $345 \mathrm{GHz}$, even in a rather coarse beam (which dilutes the signal). The optical depth maximum is found between 0 and $1 \mathrm{hr}$ and fairly close to the location where the submillimeter spectral index $\left(\alpha_{\mathrm{b} 7}^{\mathrm{b} 9}\right.$ and $\left.\alpha_{\mathrm{b} 7}\right)$ is minimum, thus in agreement with an interpretation of the spectral index variations in terms of optical depth effects, rather than genuine variations in the characteristic size of the underlying dust grain populations. The coincidence with the northern ansa is intriguing: it is suggestive of a limb-brightening effect, which implies that the outer disk is resolved by the band 7 beam. An alternative interpretation is that the temperature decrement seen in Figure 3(c), at $\sim 0.5 \mathrm{hr}$ (see also Section 4.3), should correspond to shallower slopes for optically thick emission. 

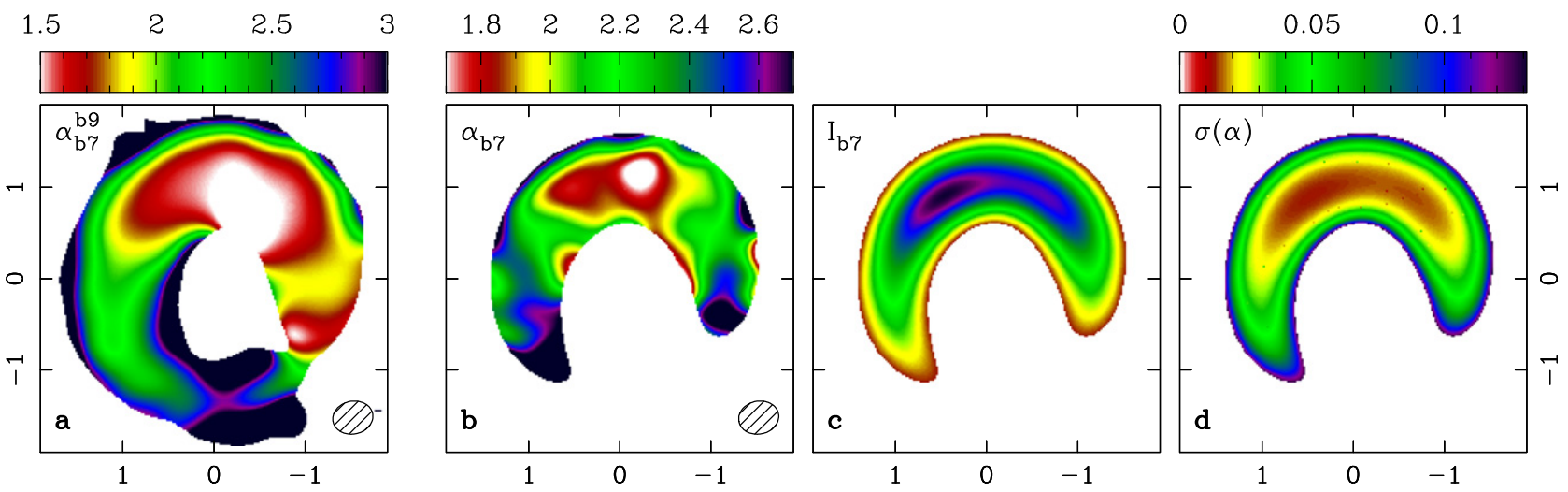

Figure 2. Spectral index maps from the ALMA band 7 and band 9 data sets. (a) Spectral index from the comparison between band 7 and band $9, \alpha_{b 7}^{b 9}$. The east-west asymmetry is suggestive of imperfections in the alignment procedure. (b)-(d) Power-law fits to the intraband 7 ALMA data, so using the four spectral windows over a frequency lever of $0.13 \mathrm{GHz}$ from the data set ALMA\#2011.0.00318.S (Fukagawa et al. 2013): (b) spectral index map $\alpha_{\mathrm{b} 7}$, (c) best-fit amplitude at the reference frequency of $345 \mathrm{GHz}$, and (d) thermal uncertainty on the spectral index map.

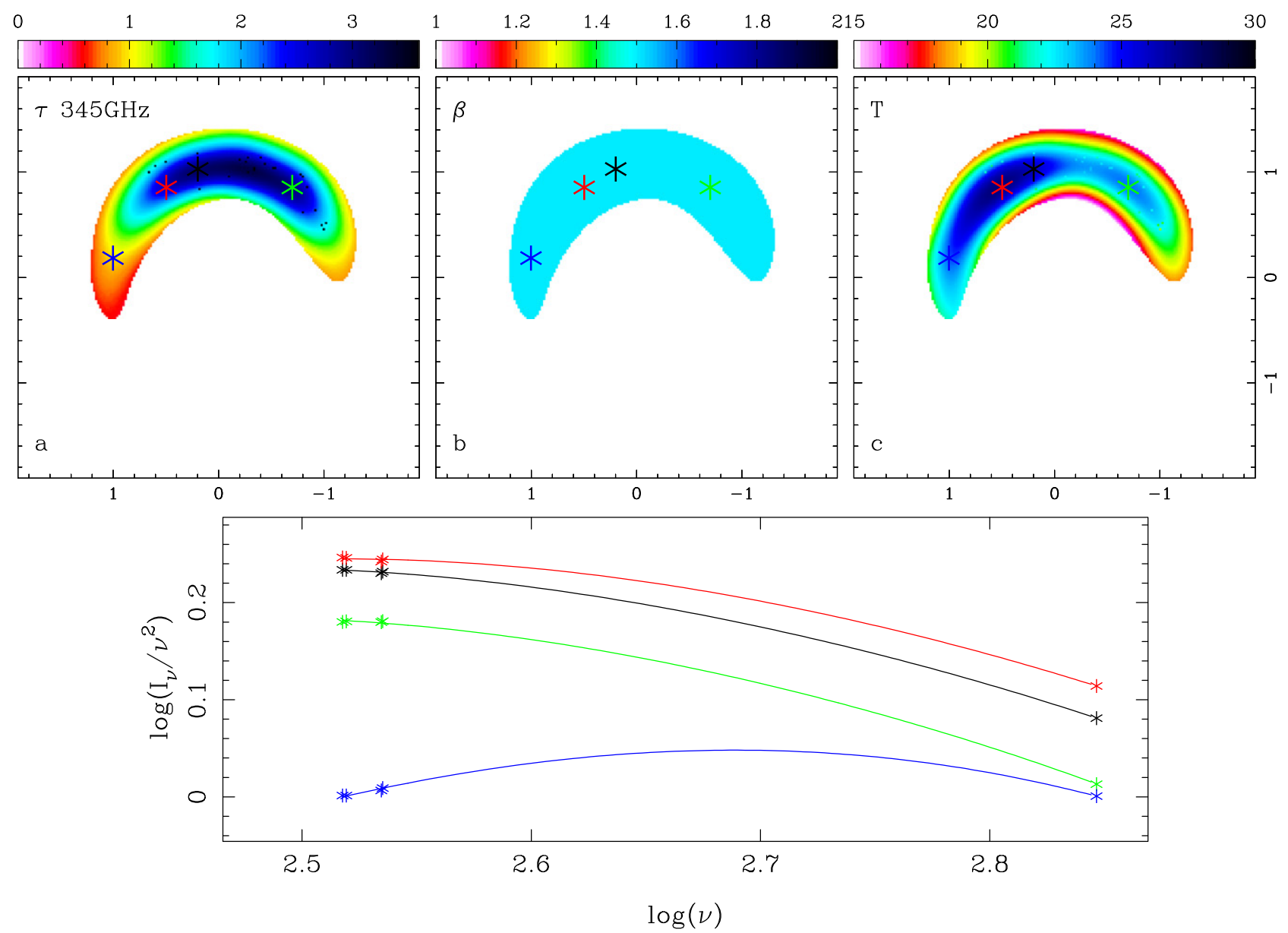

Figure 3. Spectral properties of the submillimeter continuum obtained with graybody fits to the band 9 and band 7 data (and including the intraband 7 slopes). Upper panels: (a) optical depth map at $345 \mathrm{GHz}, \tau_{\circ}(\boldsymbol{x})$; (b) flat emissivity law, taken here as $\beta=1.5$ (this plot allows us to better locate the asterisks that indicate the locations of the example spectra); (c) temperature map $T_{s}(\boldsymbol{x})$. Lower panel: example SEDs extracted at selected positions, indicated by asterisks in the upper panel. The $y$ axis shows specific intensity normalized by a $\nu^{2}$ spectrum, in arbitrary units.

Another consequence of the high submillimeter optical depths is that the double-peaked crescent morphology, best seen in band 9, reflects structure in the temperature field rather than in the density field inside the dust trap. The local band 9 emission maxima, at 10.5 and $1.5 \mathrm{hr}$, are separated by a minimum in temperature at $0.5 \mathrm{hr}$.

\section{ATCA/ALMA MULTIFREQUENCY RESULTS}

\subsection{A $34 \mathrm{GHz}$ Clump Embedded in the Submillimeter Crescent}

Resolved ATCA observations of HD 142527 in the optically thin $34 \mathrm{GHz}$ continuum reveal structure inside the submillimeter-opaque crescent, as shown in Figure 4 (see also Figure 6). 


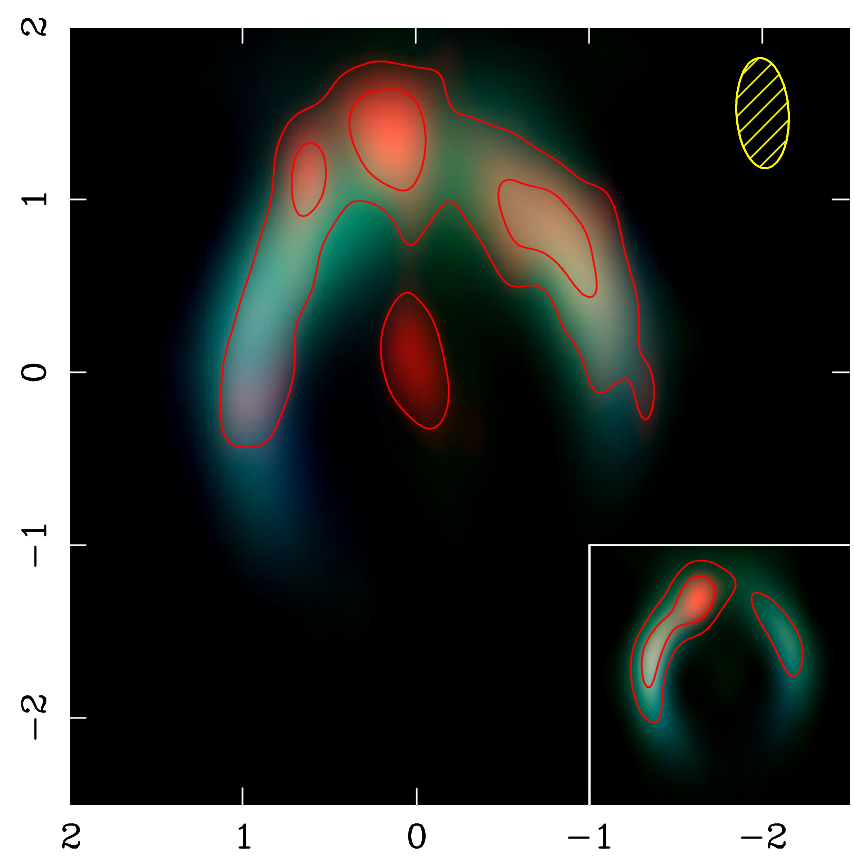

Figure 4. Multifrequency continuum highlighting compact $34 \mathrm{GHz}$ emission at $11.5 \mathrm{hr}$. The data have been filtered for the ATCA response. The RGB colors correspond to the ATCA image in red (with corresponding contours at 0.4 and 0.7 times the peak intensity at $147 \mu \mathrm{Jy}^{\text {beam }^{-1}}$ ), the ALMA band 7 in green, and the ALMA band 9 in blue (see Figure 8 for a panel with the same images). The inset shows emergent intensities predicted from the dust trap model, after filtering for the ATCA response, with $34 \mathrm{GHz}$ in red contours at 0.4 and 0.7 times the peak. The side of the inset corresponds to $3.5 \operatorname{arcsec}$.

The lower levels at $34 \mathrm{GHz}$ follow the Rayleigh-Jeans extension of dust seen in ALMA band 9. However, the peak signal stems from clumpy emission at $\sim 11.5 \mathrm{hr}$ and a longer arc at 1 to $2 \mathrm{hr}$. Both regions coincide fairly closely with the molecular decrements documented by Casassus et al. (2013, their supplementary information; see also Appendix $\mathrm{C}$ in this work). Given the thermal noise in the field, the $11.5 \mathrm{hr}$ clump is significant at $10 \sigma$, and it is robust against $u v$ filtering biases. To test for interferometer filtering artifacts, we ran Monte Carlo simulations of ATCA observations on a deconvolved model image of the band 7 data (see Appendix B), thus bringing the multifrequency data to an approximately common Fourier basis. Figure 4 shows that the ATCA clump stands out compared to the filtered ALMA maps.

The peak $34 \mathrm{GHz}$ intensity at $11.5 \mathrm{hr}$ reaches $147 \mu \mathrm{Jy}_{\text {beam }}{ }^{-1}$, with a 0 ." $64 \times 0$ ". 30 beam, and has an equivalent brightness temperature of only $0.5 \mathrm{~K}$, so the signal is optically thin (or else it is beam-diluted). For the fiducial density of water, and for a single grain size of $1 \mathrm{~cm}$ with a circular cross section, at $20 \mathrm{~K}$, we find that the observed flux density from the unresolved $11.5 \mathrm{hr}$ clump corresponds to $>7.4 M_{\oplus}$ of dust. The inequality accounts for the possibility of locking more mass in grains of different sizes that do not emit appreciably at $34 \mathrm{GHz}$.

A compact signal coincident with the stellar position is conspicuous in the ATCA map. Its spectral index at ATCA frequencies is $\alpha_{20}^{45}=1.0 \pm 0.2$, which extrapolates to the level of the faint circumstellar signal seen in ALMA band 7 (Casassus et al. 2013; Fukagawa et al. 2013). These spectral index values are consistent with free-free emission associated with a stellar wind or with stellar accretion.

\subsection{Evidence for Dust Trapping}

In another application of the graybody diagnostics, we computed the optical depth and opacity index $\left(\beta_{S}\right)$ maps corresponding to the ATCA and ALMA data sets shown in Figure 4. The resulting optical depth map shown in Figure 5 confirms the levels at $345 \mathrm{GHz}$ inferred from the ALMA band 7 and band 9 comparison. The ATCA clump at $11.5 \mathrm{hr}$ translates to a minimum in $\beta_{S}(\boldsymbol{x})$ (Figure 5(b)). This is an indication that dust grains in this clump are larger than in the rest of the crescent.

The opacity index variations account for the ATCA/ALMA morphological differences and should thus be significant at the same level. We estimated the uncertainties on the opacity index $\beta_{S}(\boldsymbol{x})$ by varying the ATCA intensities by $\pm 1 \sigma$, where $\sigma$ is the scatter in the dirty map of image synthesis residuals. As shown in Figure 5(c), the peak at $34 \mathrm{GHz}$, under the $11.5 \mathrm{hr}$ clump, corresponds to $\beta_{S}=1.50 \pm 0.03$, whereas the average value outside the regions of low $\beta_{S}$ is 1.81 , with an rms dispersion of 0.076 and typical values varying from $1.8 \pm 0.04$ at $1.5 \mathrm{hr}$ up to $1.94 \pm 0.05$ at 0.5 and $9.5 \mathrm{hr}$. Compared to the average value of 1.81 , the minimum in $\beta_{s}$ under the $11.5 \mathrm{hr}$ clump is significant at $\sim 10 \sigma$.

The temperature map is essentially fixed by the band 9 spectral point; we note a minimum in temperature toward $0.5 \mathrm{hr}$, which is also seen when using the ALMA data alone. The similarities between the temperature maps in Figures 3 and 5 are surprisingly good, considering the differences in $u v$ coverage and the different treatments for $\beta$.

\section{PREDICTIONS BASED ON A SYNTHETIC DUST TRAP MODEL}

\subsection{Biases of the Graybody Diagnostics}

The graybody line-of-sight diagnostic is based on twodimensional sky maps for $\beta_{S}(\boldsymbol{x})$ and $T_{S}(\boldsymbol{x})$, whereas the actual physical conditions are three-dimensional. Since the crescent is optically thick in band 9, the temperature is mainly set by the higher frequencies and probably overestimates the temperature in regions closer to the midplane. Hence the actual optical depths could be higher than inferred from graybody diagnostics. In order to assess biases and test the dust trap interpretation, we applied the same observational diagnostic, based on the graybody fits, to synthetic radiative transfer predictions of a parameterized dust trap model meant to approximate the phenomenon observed in HD 142527. The synthetic model was implemented in RADMC3D (Dullemond et al. 2015) and centers the dust trap at $11.5 \mathrm{hr}$. At the native resolution of the radiative transfer, the graybody optical depth map reproduces the input optical depths very closely. However, both optical depth and temperatures are biased downward by $\sim 50 \%$ after filtering by the ATCA response. The location of the minimum in the graybody opacity index, $\beta_{S}(\boldsymbol{x})$, coincides with the input dust trap, which is fairly optically thick in band 7 .

\subsection{Origin of the Molecular Decrements}

The optical depths in the synthetic trap are not deep enough and do not have the structure required to account for the observed deep molecular decrements. In particular, the existence of decrements in $\mathrm{CO}(2-1)$ (Perez et al. 2015), so at frequencies with lower optical depths, suggests that there is 

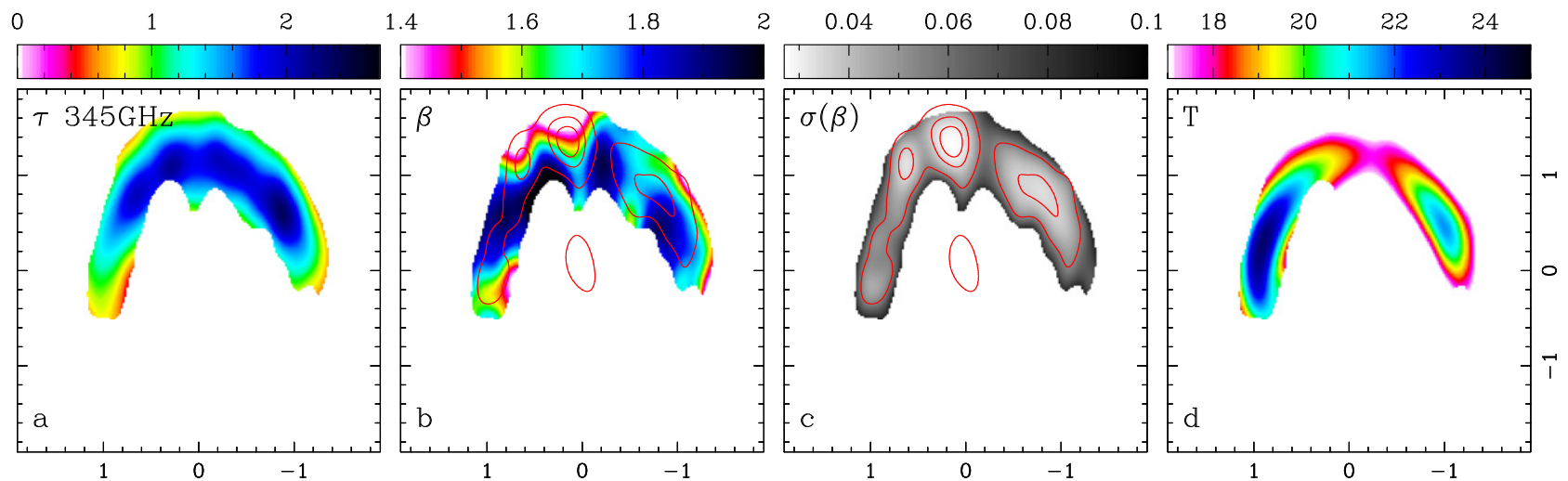

Figure 5. Optical depth maps derived from the ATCA/ALMA multifrequency data. The emissivity minimum at $11.5 \mathrm{hr}$ indicates the presence of larger grains. (a) Optical depth map at the reference frequency of $345 \mathrm{GHz}$. (b) Line-of-sight emissivity index map $\beta_{s}(\boldsymbol{x})$, with ATCA-specific intensity contours in red. (c) Root mean square uncertainties on the emissivity index map. (d) Line-of-sight temperature, $T_{s}(\boldsymbol{x})$.

ample room for other effects. Their properties could be reproduced by prescribing gas temperatures cooler than the dust, which is rather surprising (Appendix C). The decrements may also correspond to structural features of the disk, that is, shadowing in lower scale heights under the $34 \mathrm{GHz}$ clumps, due to midplanes cooler than in the rest of the outer disk at the same stellocentric radius. A mechanism for such a local "pinching" of the outer disk may be found in the dust trap scenario. In radiative equilibrium, the larger grains tend to be cooler (e.g., Aikawa \& Nomura 2006), so hydrostatic scale heights will be lower under the dust trap. The shadowed $\mathrm{CO}$ is cooler or possibly frozen out and condensed onto grain surfaces.

\subsection{Temperature Decrement Due to Shadowing on the Outer Ring}

The temperature structure inferred from the band 9 observations are readily explained by the model, which includes a tilted inner disk within $20 \mathrm{AU}$, as implied by the shadows in scattered light cast on the outer disk (Marino et al. 2015). Figure 1 shows that the predicted emergent intensities in band 9 are double peaked, much like the observations. The warped structure results in shadows projected on the outer disk where the stellar heating is blocked by the inner disk at PAs of 0.5 and $6.5 \mathrm{hr}$. The similarity with the observations (Figure 3) is remarkable considering the idealizations of the synthetic disk, which assumes a circular cavity, power laws for the grain size distributions, and steadystate dust trapping prescriptions. Another idealization of the model is steady-state passive heating.

The cooling timescale is $\tau_{C}=U /\left(\sigma_{B} T^{4}\right)$, where $U$ is the internal energy per unit area and $\sigma_{\mathrm{B}}$ is the Stefan-Boltzmann constant. The condition that $\tau_{\mathrm{c}}$ must be shorter than the crossing time under shadows that cover $\sim 20^{\circ}$ at $140 \mathrm{AU}$ (Marino et al. 2015, and Figure 12) is satisfied for surface densities lower than $50 \mathrm{~g} \mathrm{~cm}^{-2}$, which is indeed the maximum surface density in the model. It is thus reasonable to expect that the double-peaked structure of the continuum emission is determined by the temperature field and that the millimeter grains are otherwise uniformly spread along the crescent. The modulation in temperature along the crescent, caused by the inner warp, impacts the thermodynamics of the disk. This modulation imparts a periodic forcing to the outer disk, whose dynamical consequences should be investigated, and that could perhaps explain the observed spiral pattern.

\subsection{Very Lopsided Disks?}

The spectral trends in HD 142527 at submillimeter frequencies can thus be accounted for with optical depth effects, such that the data so far require little trapping for grains up to $1 \mathrm{~mm}$ in size. In other words, the large submillimeter crescent (Casassus et al. 2013) could mostly reflect the gas background, with relatively inefficient trapping, so that in our model the observed contrast ratio of $\sim 30$ at $345 \mathrm{GHz}$ is accounted for with an equal contrast in the gas. Marked gaseous asymmetries with contrasts of order 10 are seen in MHD simulations of dynamical clearing by planet formation (Zhu \& Stone 2014) and could perhaps reach higher values in models incorporating dead zones with discontinuous turbulence prescriptions (Regály et al. 2012), or with enhanced cooling inside the dust trap via dust-gas coupling. Thus we propose that other submillimeter asymmetries could partly reflect the gas, perhaps even with large contrasts such as in IRS 48, and most likely in the more moderate contrasts, such as in HD 135344B and SR 21 (Pérez et al. 2014). Consequently, a trapping origin for the dust asymmetries seen in such disks may best be studied at lower frequencies, for example, $\leqslant 50 \mathrm{GHz}$ (also where emission is optically thin).

\section{CONCLUSION}

In summary, the multifrequency observations of HD 142527 are consistent with the dust trap scenario. New ALMA observations have shown that the millimeter grains are so abundant in the crescent that efforts to observe grain growth at submillimeter wavelengths are thwarted by optical thickness. Optical depth effects account for the spectral trends at ALMA frequencies, while shadowing of UV-optical radiation by the central warp explains the temperature structure of the crescent. However, at transparent centimeter wavelengths, a compact clump of grains approaching centimeter sizes is embedded within the broader crescent of smaller submillimeter-sized grains (the exact grain sizes depend on the size distributions and opacities). These phenomena are expected in the context of the aerodynamic coupling of dust grains with pressure gradients, given the physical conditions in the passively heated outer disk of HD 142527. Prospects are good for witnessing 
planetesimal formation by dust trapping with radio observations of HD 142527 at centimeter wavelengths.

We thank Cornelis Dullemond for interesting discussions and help with RADMC3D, Matthijs van der Wiel for bringing to our attention his update on the SPIRE flux density for HD 142527, and the referee for a positive report and constructive comments. This paper makes use of the following ALMA data: ALMA\# 2011.0.00465.S and ALMA\#2011.0.00318.S. ALMA is a partnership of ESO, NSF, NINS, NRC, NSC, and ASIAA. The Joint ALMA Observatory is operated by ESO, AUI/NRAO, and NAOJ. The Australia Telescope Compact Array is part of the Australia Telescope National Facility, which is funded by the Commonwealth of Australia for operation as a National Facility managed by CSIRO. Financial support was provided by Millennium Nucleus RC130007 (Chilean Ministry of Economy), and additionally by FONDECYT grants 1130949, 3140601, 3140634, and 3140393. C.M.W. acknowledges support from ARC Future Fellowship FT100100495. S.M. acknowledges CONICYT-PCHA/Magister Nacional/2014-22140628. P.R. and V.M. acknowledge support from ALMA-CONICYT grant 31120006. This work was partially supported by the Chilean supercomputing infrastructure of the NLHPC (ECM-02).

Facilities: ATCA, ALMA.

\section{APPENDIX A OBSERVATIONS}

HD 142527 , at $\sim 140 \mathrm{pc}$, is a promising laboratory to observe ongoing gas giant planet formation and confirm the existence of the dust trap phenomenon. This transition disk has a close to face-on orientation. IR observations found an inner disk, $\sim 10$ AU (Fujiwara et al. 2006) in radius and surrounded by a particularly large gap (Fukagawa et al. 2006). A disrupted outer disk (Casassus et al. 2012) beyond $140 \mathrm{AU}$ is indicative of dynamical clearing of the gap by giant planet formation. The cavity is devoid of bodies larger than $\sim 4 M_{\text {jup }}$ (Casassus et al. 2013), but an accreting companion has been reported at $\sim 10$ AU (Biller et al. 2012; Close et al. 2014; Rodigas et al. 2014; with a binary mass ratio of $0.01-0.1$ ). The bulk of the mass lies in the outer disk, whose crescent morphology (Ohashi 2008; Casassus et al. 2013) approximately anticorrelates with molecular-line emission (Casassus et al. 2013; van der Plas et al. 2014; Perez et al. 2015). A complex array of trailing spiral arms sprouts away from the outer disk (Fukagawa et al. 2006; Casassus et al. 2012; Rameau et al. 2012; Canovas et al. 2013; Avenhaus et al. 2014; Christiaens et al. 2014).

In this section we provide details on the data sets that sustain our multifrequency analysis. Appendix A.1 presents the new ALMA observations, Appendix A.2 presents the new ATCA data, and Appendix A.3 summarizes previously published ALMA data. Appendix A.4 briefly describes our image synthesis procedure, while Appendix A.5 and Appendix A.6 describe the alignment of the multifrequency ALMA data and associated spectral index trends, and Appendix A.7 describes the alignment of the ATCA and ALMA data sets.

\section{A.1. ALMA Band 9 Observations}

ALMA Band 9 observations of HD 142527 for programJAO. ALMA\#2011.0.00465. S were carried out in 2012, in the nights of June 3, from 02:52:53 to 04:39:10 UT, and June 4, from 00:43:22 to $02: 42: 26 \mathrm{UT}$. The precipitable water vapor (PWV) in the atmosphere was stable between $0.54 \mathrm{~mm}$ and $0.62 \mathrm{~mm}$ on June 3 , with a median value at zenith of $0.581 \mathrm{~mm}$. Conditions on June 4 were similar, with a median PWV at zenith of $0.597 \mathrm{~mm}$. The ALMA correlator provided $1875 \mathrm{MHz}$ bandwidth in four different spectral windows (SPWs) at $488.28125 \mathrm{kHz}$ resolution (or $211.67 \mathrm{~m} \mathrm{~s}^{-1}$ at $691.47308 \mathrm{GHz}$ ) per channel. Each spectral window was positioned in order to target the $\mathrm{CO}(6-5)$ transition at $691.47308 \mathrm{GHz}, \mathrm{HCO}^{+}(8-7), \mathrm{H}^{13} \mathrm{CO}^{+}(8-7)$, and $\mathrm{HCN}(8-7)$. Only $\mathrm{CO}(6-5)$ was detected. This line is the topic of a separate article. On June 3 , the measured system temperatures ranged from $\sim 1400 \pm 500 \mathrm{~K}$ in the spectral window covering $\mathrm{CO}(6$ 5) to $\sim 2000 \pm 700 \mathrm{~K}$ in the spectral window covering $\mathrm{HCO}^{+}$. System temperature values 100-200 K higher were recorded on June 4 . The number of $12 \mathrm{~m}$ antennas available at the time of the observation was 20 , although one antenna reported very large system temperatures (DV12) and was flagged during data reduction. Excluding calibration overheads, the total time on source for HD 142527 was 43 minutes on June 3 and 49 minutes on June 4 . The primary flux calibrator was Titan, while 3C 279 was used as bandpass calibrator. We used J1517243 as phase calibrator, for which we measure a flux density of $0.70 \pm 0.14 \mathrm{Jy}$ at $702 \mathrm{GHz}$, where the uncertainty accounts for systematic calibration errors. This measurement is consistent with historical values for this source. The radiometer phase correction errors were large enough to decorrelate the signal. We used the self-calibration algorithm to determine improved antenna-based phases that were consistent with the continuum image of HD 142527.

\section{A.2. ATCA Data}

We used ATCA to observe HD 142527 in several array configurations in May 2010 (6A), June 2010 (6C), July 2013 (6A), and August 2013 (H168), with maximum baselines extending to $6 \mathrm{~km}$. The Compact Array Broadband Backend, or CABB (Wilson et al. 2011), provides two sidebands with bandwidths of $2 \mathrm{GHz}$ in 2024 channels each. We centered each sideband at $19 / 21,23 / 25,33 / 35$, and $41 / 43 \mathrm{GHz}$ (in this work we focus on the $33 / 35$ data set). Complex gains were derived from observations of the quasar 1600-44, separated by about 2.8 degrees on the sky from HD 142527. The gain calibrator was typically observed every 5-15 minutes for between 1 and 3 minutes duration, dependent on atmospheric conditions. Pointing checks were also made on the quasar every 60-90 minutes. The bandpass response was determined from 15 minute observations of the quasar 1253-055 (3C 279). The absolute flux calibration was performed using ATCA's primary centimeter-band flux calibrator, the quasar 1934-638. All subsequent data reduction and calibration was performed with the Miriad software (Sault et al. 1995). The CABB setup at 33/ $35 \mathrm{GHz}$ was used in four runs with different array configurations, including H168, which allows us to estimate that the amount of flux loss in the extended configurations is negligible. The flux densities obtained in H168, $1.28 \pm 0.04 / 1.48 \pm 0.04$, in $\mathrm{Jy}$, for each sideband centered at $33 / 35 \mathrm{GHz}$, is indistinguishable from the values of $1.23 \pm 0.05 / 1.49 \pm 0.06,1.34$ $\pm 0.11 / 1.48 \pm 0.11$, and $1.25 \pm 0.13 / 1.24 \pm 0.09$ obtained in $6 \mathrm{C}, 6 \mathrm{C}$, and 6A, respectively.

The ATCA data with the best dynamic range were obtained in $\mathrm{Ka}$, that is, at $33 \mathrm{GHz} / 35 \mathrm{GHz}$. For efficiency we averaged 

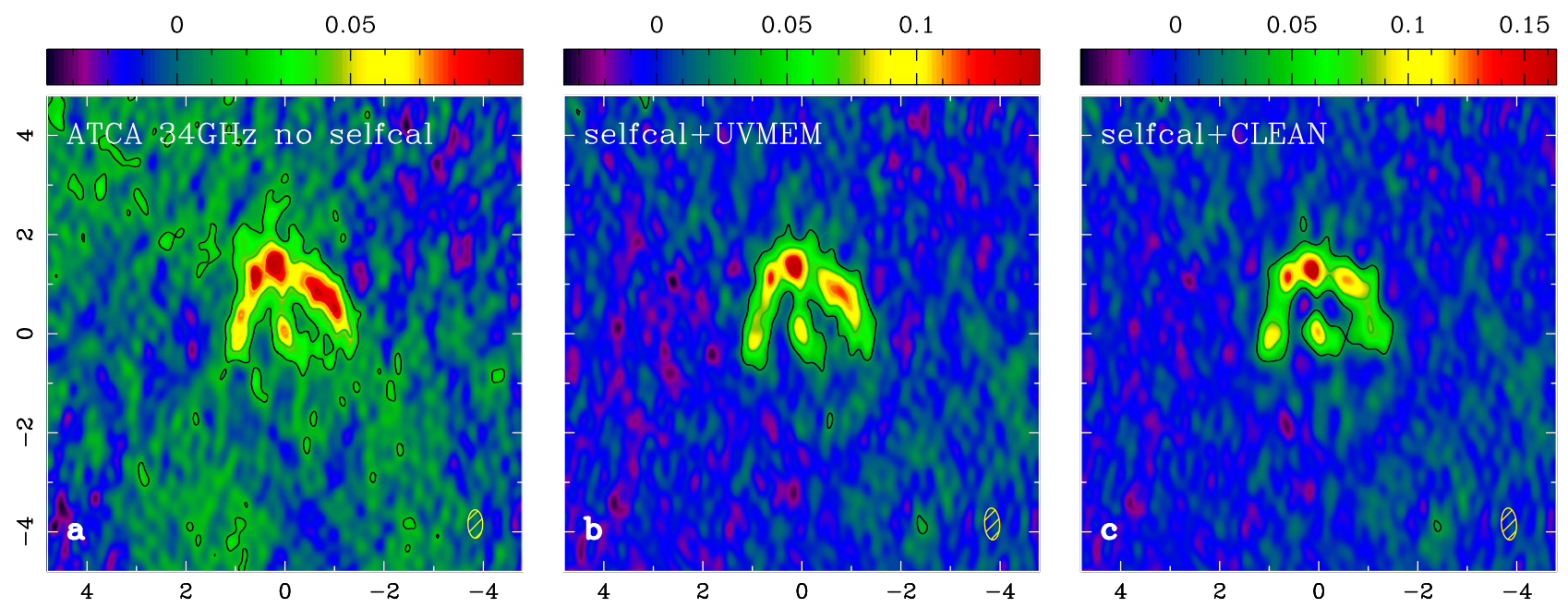

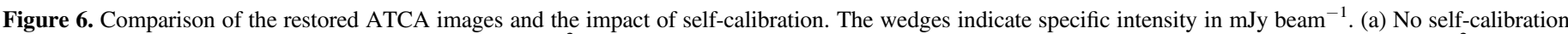

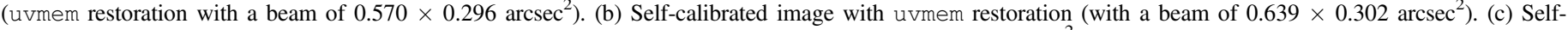
calibrated image with CASA (McMullin et al. 2007) clean restoration (with a beam of $0.644 \times 0.304 \operatorname{arcsec}^{2}$, CASA version 4.2.2).

each $\mathrm{CABB} 2 \mathrm{GHz}$ spectral window into eight channels. We then brought all of the $6 \mathrm{~km}$ visibility data in Ka to a common epoch, which we chose as 2012 July, by correcting for the source proper motion with a translation phase (using the Miriad task uvedit). The $6 \mathrm{~km}$ data in Ka were then concatenated with the task concat from CASA (McMullin et al. 2007) version 4.2.2. A standard clean restoration revealed a faint modulation at low spatial frequencies in the field, atypical of image synthesis artifacts and reminiscent of phase calibration errors. We therefore applied self-calibration of the visibility phases, using the clean model and the CASA tasks gaincal and applycal. We used a solution interval of 30 minutes and combined both SPWs for sensitivity. The clean restoration of the corrected visibilities results in essentially the same source morphology as the uncorrected data set, but with less systematics in the field. The impact of self-calibration can be assessed by inspection of Figure 6, where we show the restored images obtained with our uvmem (Casassus et al. 2006, 2013) tool before and after self-calibration (more information on this image synthesis is given in Appendix A.4).

\section{A.3. Previous ALMA Band 7 Observations}

As explained in Figure 7 and Appendix A.5, the calibrated data set ALMA\#2011.0.00465.S is affected by faint outliers. We therefore used the ALMA band 7 obtained by Fukagawa et al. (2013), ALMA\#2011.0.00318.S, keeping only the last scheduling block, observed on 2012 August 10. The reason for not combining the whole data set is because the dynamic range under the crescent is limited by image synthesis, rather than thermal noise, and because pointing uncertainties would degrade the beam if combined without a tedious alignment procedure (see Appendix A.5). For instance, we measured an offset of 0.065 arcsec between this scheduling block and the single scheduling block in band 7 from ALMA\#2011.0.00465.S, comparing exclusively the common spectral window at $342.8 \mathrm{GHz}$. These offsets are within the nominal pointing accuracy of $\sim 0.1$ arcsec. Although small, such offsets have appreciable consequences when deriving spectral index maps.

In summary, we used the data set acquired by Fukagawa et al. (2013), program JAO.ALMA\#2011.0.00318.S, but tied the astrometric pointing to the data previously presented by Casassus et al. (2013), JAO.ALMA\#2011.0.00465.S. Further details on the astrometric registrations are given in Appendices A.5 and A.7.

\section{A.4. Image Synthesis}

For image synthesis, we use a nonparametric least-squares modeling technique (Casassus et al. 2006) with a regularizing entropy term (i.e., as in the family of maximum entropy methods). We call this tool uvmem. These deconvolved model images are "restored" by convolution with the clean beam and by adding the dirty map of the visibility residuals calculated using the CASA package, for a chosen weighting scheme. In this work we used natural weights.

The clean algorithm is an efficient and widely accepted technique to produce images from radio-interferometer data. We used the standard CASA clean, that is, Cotton-Schwabb, to produce a traditional image synthesis that can be compared with our uvmem tools. A comparison between uvmem and clean is shown in Figure 6.

We assume that the relative values of the visibility weights in the ALMA data are accurate. However, the absolute values do not match the scatter in the visibility samples recorded at each integration. In order to extract statistics and perform leastsquares fits, we therefore scaled the weights so that they correspond to $1 / \sigma^{2}$, where $\sigma$ is the root mean square dispersion of all the visibility samples recorded for a given baseline and channel. This procedure was carried out using a tool we call uvreweight. A single scale factor was applied to the weights of the whole data set, given by the median values of the ratios between observed scatter and tabulated weights. Thus we respected the relative values of the weights as provided in the calibrated data set. We used median averages for a robust estimate of the observed scatters, after correction by the median absolute deviation. In this uvreweight-processed data, the absolute value of the weights can be used to extract statistics.

\section{A.5. Alignment of the Multifrequency ALMA Data}

We aligned the multifrequency interferometer data by crosscorrelating the visibilities, as illustrated in Figure 7. We first 

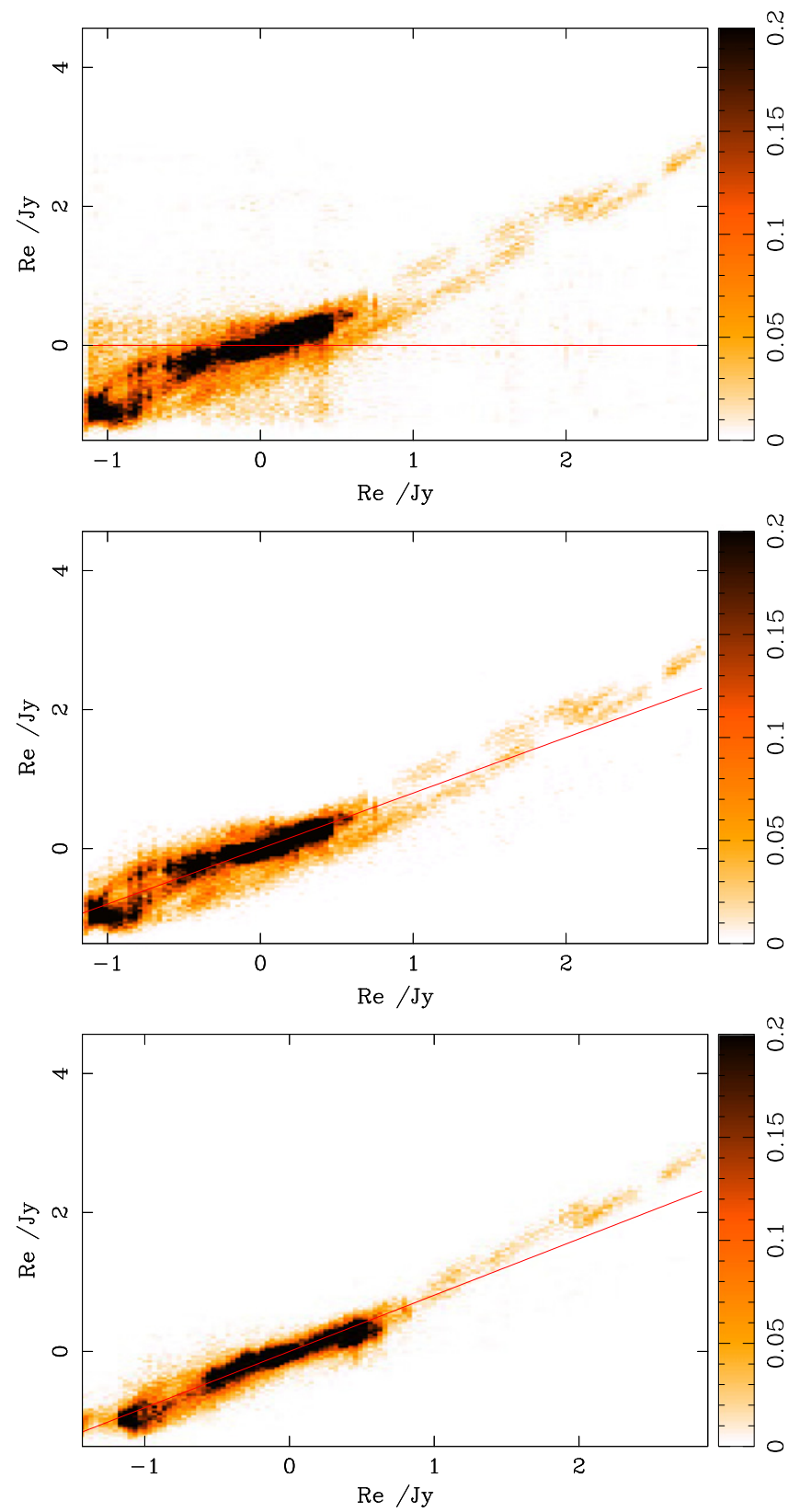

Figure 7. Band 7 vs. band 9 visibility scatter plot, from data set 2011.0.00465.S. Top: cross-correlation before outlier filtering. Middle: after outlier filtering, but before astrometric WCS correction. Lower plot: after the alignment procedure described in Appendix A.5. In this case, the applied WCS offset is $\left(-0 .{ }^{\prime \prime} 063,-0\right.$. . 016$)$ along R.A. and decl. We see here that this band 7 data set ALMA\#2011.0.00465.S is affected by faint outliers; this is why we used the data from ALMA\#2011.0.00318.S (Fukagawa et al. 2013). Since the dynamic range under the crescent is not limited by thermal noise, we kept only the last scheduling block, observed on 2012 August 10, to minimize pointing offsets. For consistency we tied the astrometric pointing to $2011.0 .00465 . \mathrm{S}$.

approximately bring two independent visibility data sets to a common $u v$ coverage in the following way. We select as reference the data set with the more compact $u v$ coverage (usually the lower frequency), and we produce a deconvolved model using uvmem of the visibility data set with the more extended $u v$ coverage. We then compute synthetic interferometer data corresponding to the more compact $u v$ coverage using our tool uvsim. For consistency, the procedure is also applied to the reference. Having produced comparable visibility data sets, we regularly sample shifts in the world coordinate systems (WCS) of the two frequencies in a uniform grid of positional offsets $\left\{\boldsymbol{\delta}_{i}\right\}$ (assuming that the parallactic calibration is perfect in both cases). For each WCS offset $\boldsymbol{\delta}_{i}$ we computed shifted band 9 visibilities:

$$
V_{b 9}^{\text {shift }}\left(\boldsymbol{u}_{k}\right)=V_{b 9}\left(\boldsymbol{u}_{k}\right) \exp \left[i 2 \pi\left(\boldsymbol{\delta}_{i} \cdot \boldsymbol{u}_{k}\right)\right] .
$$

We then calculated a linear regression between the real and imaginary parts of visibilities, used as independent data, and computed the $L_{2}$ distance (squared norm) between model and data. We form an image $\chi^{2}\left(\left\{\delta_{i}\right\}\right)$ of the $L_{2}$ distances for each $\boldsymbol{\delta}_{i}$. The best WCS shift is given by the centroid $\boldsymbol{\delta}_{\circ}$ of an elliptical fit to $\chi^{2}\left(\left\{\delta_{i}\right\}\right)$. Although we measure the shifts $\boldsymbol{\delta}_{\circ}$ in the $u v$ plane, the actual application of $\delta_{\circ}$ to bring images to a common WCS system is carried out after image restoration, so in the image plane.

\section{A.6. Spectral Index Maps}

Thanks to the positional alignment procedure, we can build a spectral index map between band 7 and band 9 by bringing both data sets to an approximately common $u v$ coverage, as outlined in Appendix A.5. The spectral index map $\alpha_{\mathrm{b} 7}^{\mathrm{b}}$, taken on images at 329.3 and $702 \mathrm{GHz}$ restored with the $329.3 \mathrm{GHz}$ beam, is shown in Figure 2(a). We caution that the absolute values of the spectral index map $\alpha_{\mathrm{b} 7}^{\mathrm{b} 9}$ are dependent on the chosen absolute flux calibration and spectral energy distribution (SED), as explained in Appendix D. However, the relative variations in $\alpha_{\mathrm{b} 7}^{\mathrm{b} 9}$ are independent of the chosen flux scale. This interband spectral index map is likely affected by imperfect alignment.

In order to build a spectral index map based on the intraband frequency lever of the ALMA band 7 data, we selected the lowest-frequency spectral window as reference $u v$ coverage. For instance, in the spectral fits to the intraband SPWs of the ALMA band 7 data ALMA\#2011.0.00318.S, this corresponds to $329.3 \mathrm{GHz}$. We then bring all four SPWs in band 7 to a comparable $u v$ coverage by following the procedure outlined in Section A.5 (so the reference SPW was also processed in the same way as the other three SPWs).

Once all four SPWs are brought to a comparable $u v$ coverage, we extract a spectral index map by fitting the restored specific intensity maps with a power law. There are therefore two free parameters per pixel, the intensity at the reference frequency, and the power-law exponent. Figure 2 shows the resulting fit, along with the $1 \sigma$ error on the spectral index. We caution that the absolute values of this intraband spectral index map depend on the choice of flux scale, as explained in Appendix D. However, the morphological trends are independent of the absolute flux scale.

\section{A.7. Alignment of the ATCA and ALMA Data}

The offset between the ATCA and the ALMA band 7 data from ALMA\#2011.0.00465.S was within our measurement errors. Since in the ATCA data we have two intermediate frequencies (IFs) centered on 33 and $35 \mathrm{GHz}$, we can test the alignment by cross-correlating each IF separately, as in Appendix A.5, and also by requiring consistency between the real and imaginary parts. It turned out that the ATCA/band 7 offset is systematically smaller than 0 " 15 and changes sign. We concluded that the ATCA 

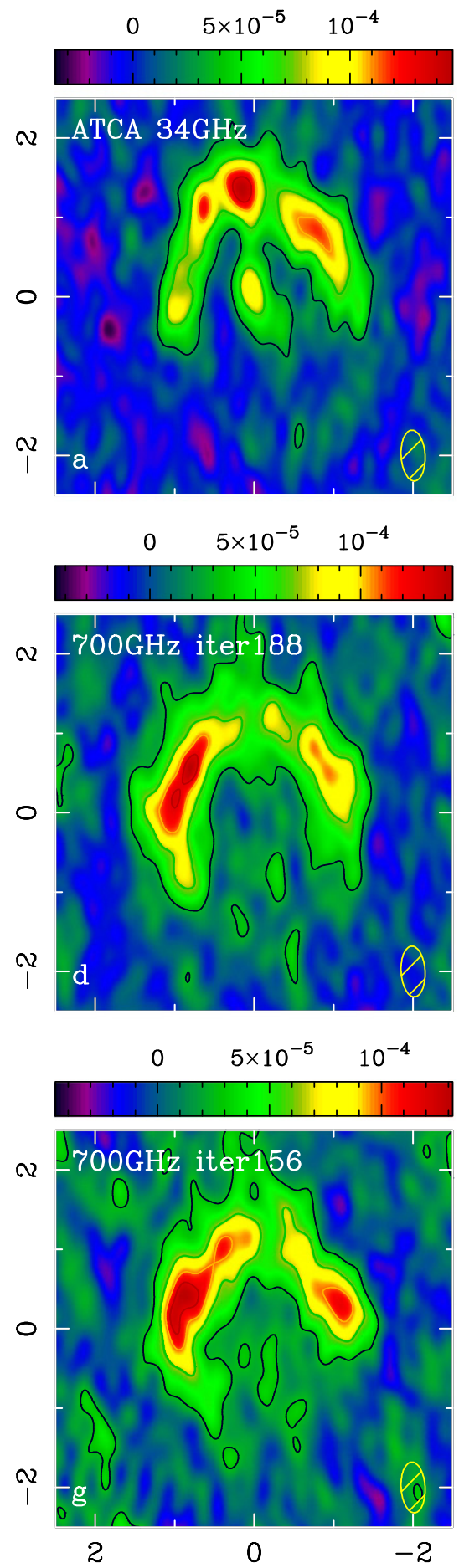
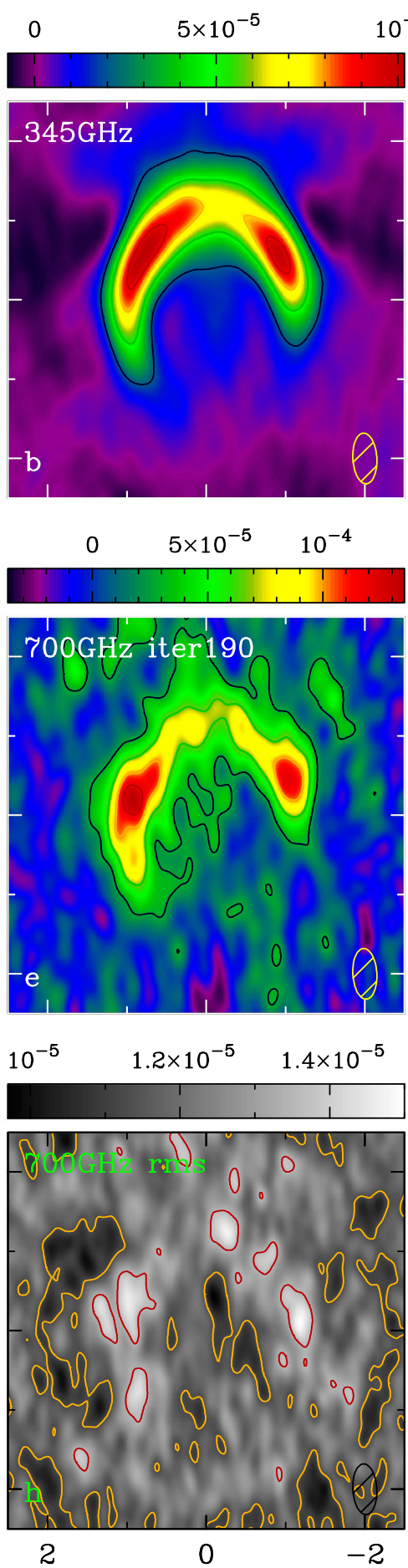
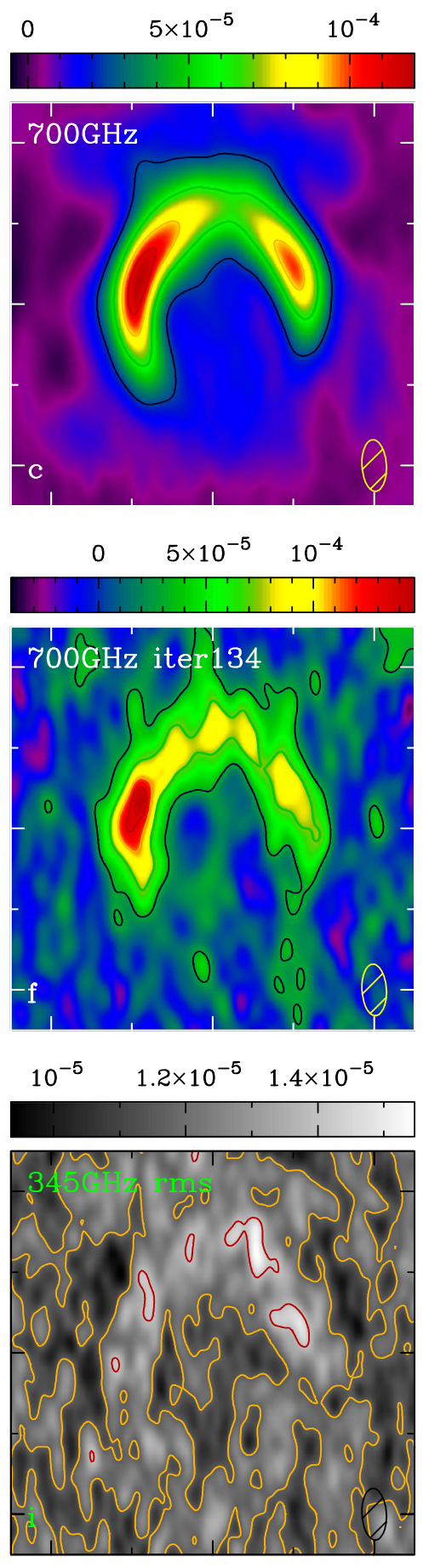

2

$-2$

Figure 8. Statistics of the multifrequency morphological differences inferred from MC simulations. All images have been restored from uvmem models. (a) Restored ATCA image at $34 \mathrm{GHz}$. (b) Average of ATCA observations with 200 realizations of noise on the band 7 data. (c) Average of the MC simulations for band 9. (d)-(g) Different realizations of noise. (h) rms scatter of the band 9 simulations. (i) rms scatter of the band 7 simulations.

and ALMA pointings are consistent, but that the morphologies are too different for a more refined alignment with our cross-correlation scheme. We also attempted the standard strategy of identifying a common anchor point, in this case the stellar position. We document this exercise as it bears on the morphology of the signal from the star's vicinity in ALMA band 7. The central free-free signal, at the limit of dynamic range in band 7, is difficult to use as an astrometric reference because its morphology is sensitive to self- calibration and it varies from a compact inner disk reported by Fukagawa et al. (2013) to a gap-crossing filament in our previous analysis (Casassus et al. 2013). However, the continuum filament we image in our self-calibrated band 7 data, after filtering for outliers using our tool uvreweight, culminates in a local maximum near the central star, now in closer agreement with Fukagawa et al. (2013). Given the uncertainties affecting the very faint stellar signal in band 7 , the default astrometries for ATCA and ALMA band 7 


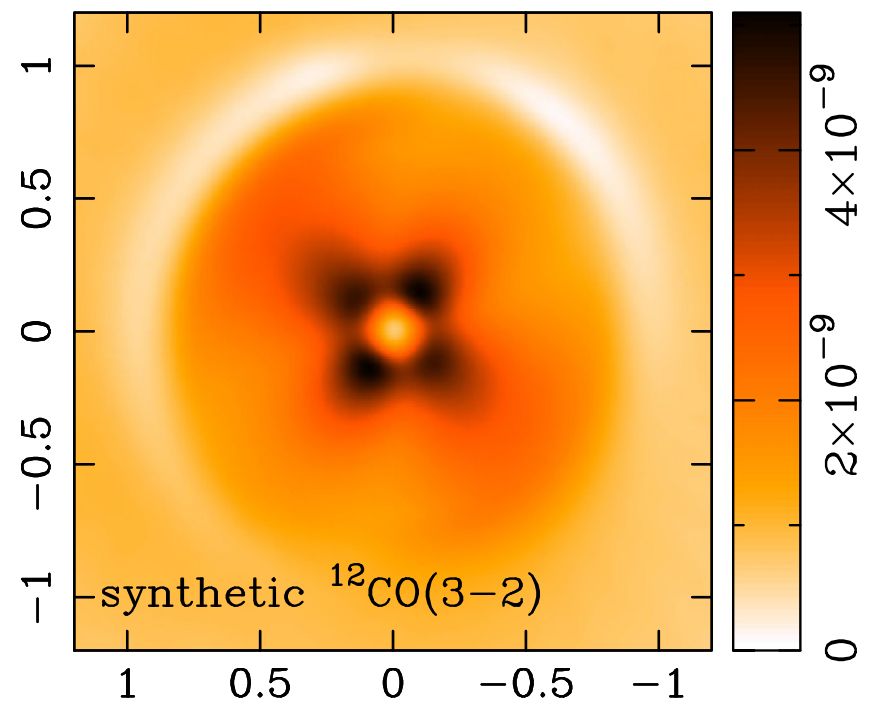

Figure 9. Frequency-integrated image of the ${ }^{12} \mathrm{CO}(3-2)$ emission calculated with RADMC3D on our preferred model for the dust trap of HD 142527, after continuum subtraction. The $x$ and $y$ axes indicate offset from the stellar position along R.A. and decl., in arcsec.

ALMA\#2011.0.00465.S appear to be consistent. We note, however, that the $11.5 \mathrm{hr}$ emission appears to extend outward in radius, by about $0.3 \pm 0$ ! 1 , compared to ALMA band 7 , while the $1 \mathrm{hr}$ clump does not. But given the above pointing uncertainties, this effect is not significant enough to warrant further consideration and modeling. We brought the multifrequency data of HD 142527 to a common $u v$ coverage following the same procedure as for the multifrequency ALMA data. The main difference is that here we used the ATCA data as reference. The resulting restored images are shown in Figure 4, where the origin of coordinates corresponds to the location of HD 142527 at the 2012 June epoch. The central point source at $34 \mathrm{GHz}$ is satisfactorily aligned with the origin.

\section{APPENDIX B \\ MONTE CARLO TESTS FOR THE MULTIFREQUENCY MORPHOLOGICAL DIFFERENCES}

Images obtained with pre-ALMA radio interferometry may be affected by $u v$-coverage artifacts. Despite the efforts invested in covering the $u v$ plane in several ATCA runs, with different array configurations, it is important to test for such $u v$ coverage artifacts, which could, for instance, inject the $11.5 \mathrm{hr}$ local maximum. We first produce deconvolved model images of the ALMA band 7 and band 9 visibility data using our tool uvmem, and then we simulate ATCA observations on these model images, following exactly the same $u v$ coverage as in the ATCA $34 \mathrm{GHz}$ concatenated data using our tool uvsim. The simulated visibilities are then scaled by a linear regression (forced to cross the origin) so that the simulated and ATCA visibilities are comparable in magnitude. Following this scaling, we inject thermal noise given by the observed visibilities weights (having first applied our tool uvreweight to replace the ATCA visibility weights by the observed scatter). We repeat this procedure 200 times before taking statistics.

Figure 8 summarizes the result of these Monte Carlo (MC) simulations. We see that the signal is more extended in the ALMA bands than it is in ATCA, so the visibility scaling we applied results in a lower signal-to-noise ratio than in the actual

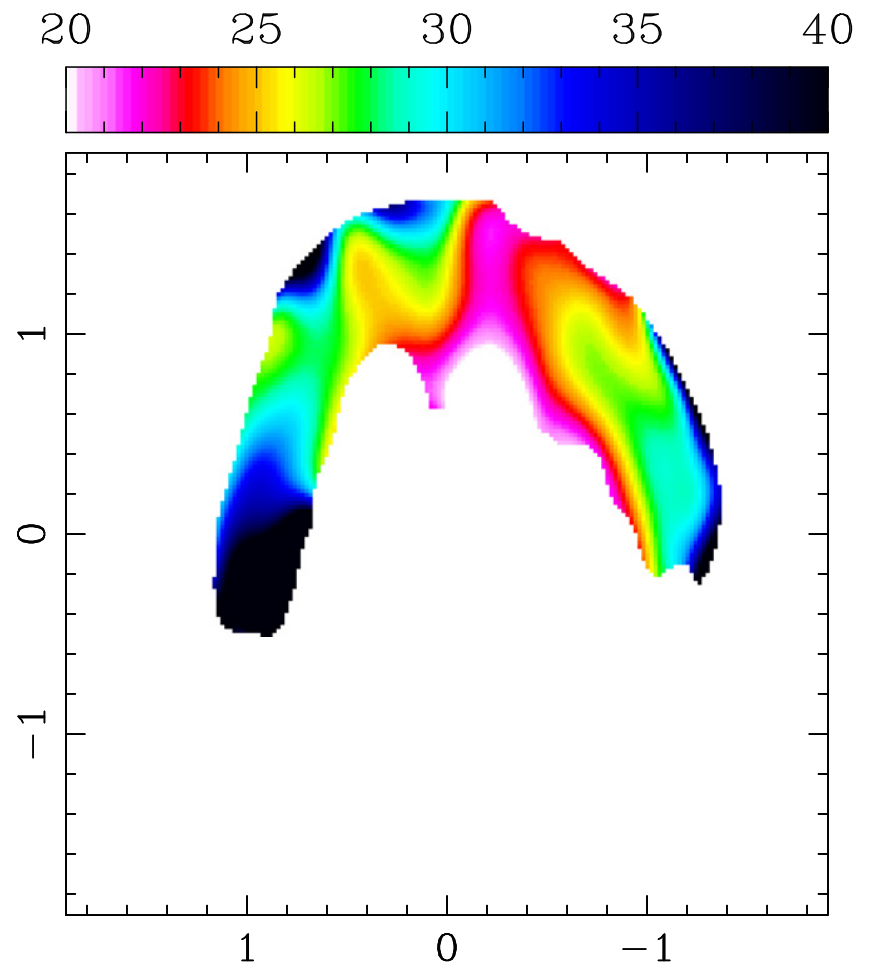

Figure 10. Temperature map resulting from a steeper submillimeter spectral index slope than suggested by the observations. We plot the graybody line-ofsight temperature for the alternative flux scale discussed in Appendix D, showing that the resulting temperature map is more irregular than Figure 5.

ATCA observations. In this sense our MC simulations are conservative because we are injecting more noise relative to the signal (by about $40 \%$ in band 7). The final rms dispersions are up to $\sim 1 / 7$ of the differences between the ATCA and ALMA images; in other words, the differences between the ATCA and ALMA images range from $-3 \sigma$ to $+6.2 \sigma$. The reduced $\chi^{2}$ taken over pixels where both ATCA and ALMA are above $1 / 3$ of the peak is 4.2 for band 7 , and 4.9 for band 9 , for 12 independent data points (the number of ATCA beams enclosed in the 0.3 times maximum contour); the images are thus different with a very high confidence level. In particular, under the $11.5 \mathrm{hr}$ clump (so at the ATCA maximum), the difference from the band 7 average is 5.9 times the band $7 \mathrm{rms}$.

An additional test was performed by subtracting the synthetic band 7 ATCA visibilities from the $34 \mathrm{GHz}$ ATCA data, after appropriate scaling by linear regression. The stellar emission stands out in the residuals, as well as the $11 \mathrm{hr}$ ATCA clump. These significant residuals, both positive and negative, point to genuine morphological differences between $34 \mathrm{GHz}$ and $345 \mathrm{GHz}$. In particular, the peak residual corresponding to the $11 \mathrm{hr}$ clump is at $50 \mu \mathrm{Jy}$ beam $^{-1}$, while the thermal noise is at $10 \mu \mathrm{Jy}_{\text {beam }}{ }^{-1}$.

\section{APPENDIX C COINCIDENCE BETWEEN ATCA MAXIMA AND MOLECULAR LINE DECREMENTS}

The $34 \mathrm{GHz}$ clumps coincide with the molecular-line decrements in $\mathrm{HCO}+(4-3)$ and in $\mathrm{CO}(3-2)$ that have been previously documented (Casassus et al. 2013). It has been suggested that an optically thick continuum may somehow be responsible for the correlation of the ${ }^{12} \mathrm{CO}$ decrements with the $345 \mathrm{GHz}$ crescent (Casassus et al. 2013; van der Plas 


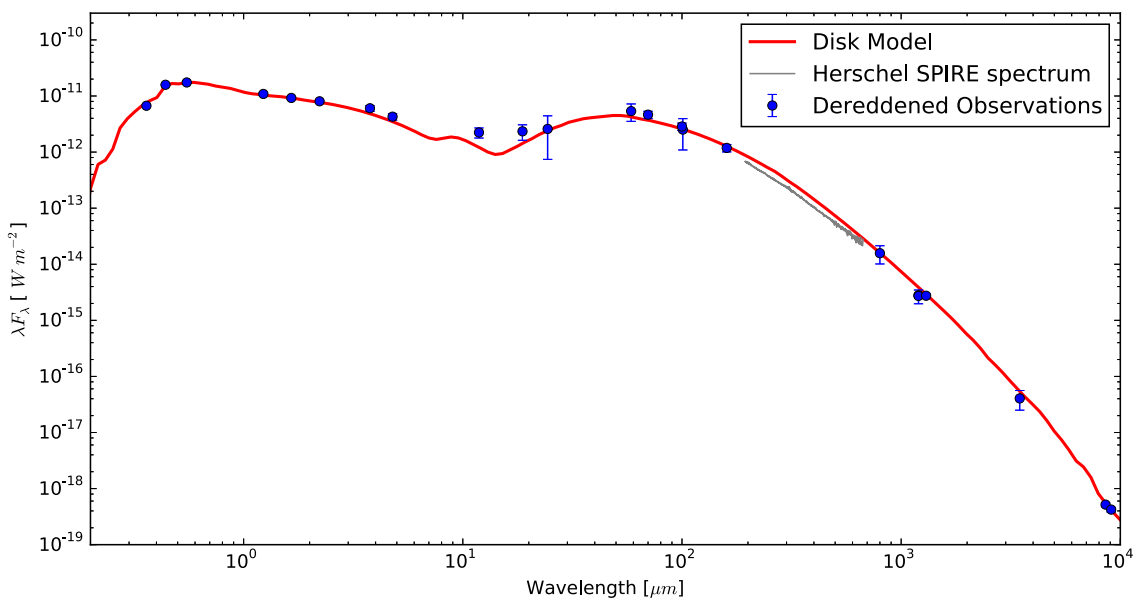

Figure 11. Observed and model SED for HD 142527.

et al. 2014). Indeed, as shown in Figure 9, the synthetic dust trap model documented below readily reproduces such decrements with a dust-to-gas mass ratio of 94, a standard $\mathrm{CO}$ abundance of $10^{-4}$, and the following temperature profile:

$$
\begin{gathered}
T=70 \mathrm{~K}, \text { if } r<30 \mathrm{AU}, \text { or } \\
T=70 \mathrm{~K}\left(r /(30 \mathrm{AU})^{-0.5} \text { if } r \geqslant 30 \mathrm{AU} .\right.
\end{gathered}
$$

However, setting the gas temperature to a representative dust species in the outer disk attenuates the depth of the decrements: they are restricted to the outline of the underlying optical depth field. The decrements are reproduced by the above prescription for the gas temperature because the gas temperatures in the crescent, at $r=140 \mathrm{AU}$, are $\sim 30 \mathrm{~K}$ and cooler than the continuum temperatures in the model, which reach 50 to $60 \mathrm{~K}$. Thus, in this case, the gas acts like a cold foreground on a hotter continuum background, which after continuum subtraction appears like foreground absorption. This possibility entails many questions: $\mathrm{CO}$ at higher altitudes could be hotter than the background continuum, so it could still stand out after the subtraction of a colder optically thick continuum. Yet the gas temperature prescription above is inspired by the molecular line data; higher temperatures can be ruled out as they greatly overestimate the integrated $\mathrm{CO}$ line fluxes.

\section{APPENDIX D ABSOLUTE FLUX SCALE}

The inferred graybody diagnostics and spectral index maps depend on the absolute calibration of the input multiwavelength data. There are, however, substantial uncertainties on the observed SED. For instance, the flux densities reported for different ALMA programs in band 7 vary from 2.8 to $3.45 \mathrm{Jy}$ at $342.9 \mathrm{GHz}$, so by $\sim 20 \%$ (Casassus et al. 2013; Fukagawa et al. 2013). The band 9 flux densities are affected by similarly large uncertainties. We adopted a reference band 7 flux density of $3.1 \mathrm{Jy}$ at $342.9 \mathrm{GHz}$ and used the SPIRE flux density (van der Wiel et al. 2014) for band 9, so $12.0 \mathrm{Jy}$ at $702 \mathrm{GHz}$. The comparison between the SPIRE flux density and the band 7 levels implies a spectral index of $\alpha_{\mathrm{b} 7}^{\mathrm{b} 9} \sim 1.9$. On the other hand, the band 6 ALMA data yields $\alpha_{\mathrm{b} 6}^{\mathrm{b} 7} \sim 2.5$ (with $1.08 \mathrm{Jy}$ at 225.4 GHz; Perez et al. 2015), so we assumed that a plausible value for the intraband 7 spectral index is $\alpha_{\mathrm{b} 7}=2.2$.

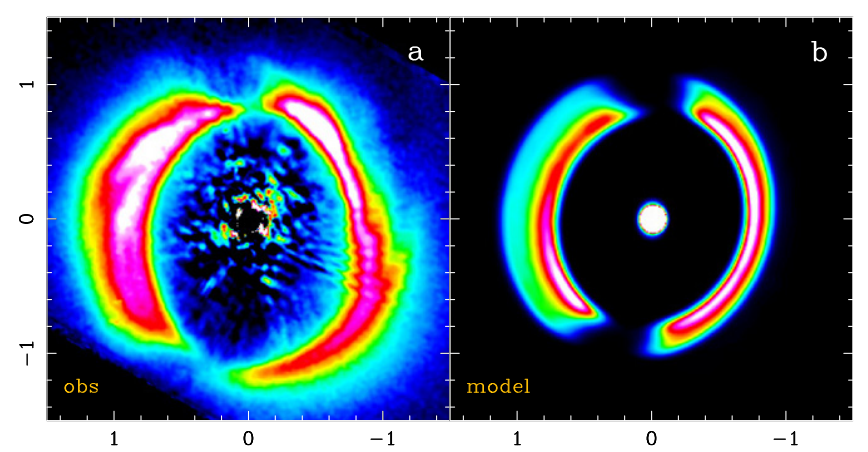

Figure 12. Consistency of the synthetic dust trap model with the observed scattered light shadows. (a) NACO-PDI H-band image from Avenhaus et al. (2014). (b) Radiative transfer prediction in H-band polarized intensity from the dust trap model (after Gaussian smoothing, 70 mas FWHM).

We investigated other possible SEDs at the limits imposed by the observational constraints. For instance, we brought the band 9 flux density up to $15.7 \mathrm{Jy}$ at $700 \mathrm{GHz}$, while decreasing the band 7 flux density to $2.17 \mathrm{Jy}$ at $342.9 \mathrm{GHz}$. In this case, the morphological trends in $\tau_{\circ}$ and $\beta_{S}$ remained, but the peak optical depths at $345 \mathrm{GHz}$ dropped by a factor of $\sim 2$. However, the temperature map appears more irregular, as summarized in Figure 10, suggesting that this flux scale may be less realistic.

\section{APPENDIX E SYNTHETIC DUST TRAP MODEL}

We constructed a synthetic model of a protoplanetary disk with a dust trap to make predictions comparable to the ALMA and ATCA observations of HD 142527. This synthetic model matches the observed SED (see below) and is a perfected version of that which accounts for the scattered-light shadows (Marino et al. 2015) with a tilted inner disk. This model allows us to (1) assess if the observations can be interpreted with steady-state dust trapping formulae, given the tilted inner disk, and (2) estimate the biases involved in the uniform-slab graybody model.

The inner disk has a total dust mass of $2.0 \times 10^{-9} M_{\odot}(25 \%$ amorphous carbon and $75 \%$ silicates) and an aspect ratio of 0.14 . The outer disk is where the dust trap is observed, so we describe it in more detail. It goes from 115 to $300 \mathrm{AU}$ with a rounded disk wall between 115 and $140 \mathrm{AU}$ and is composed 

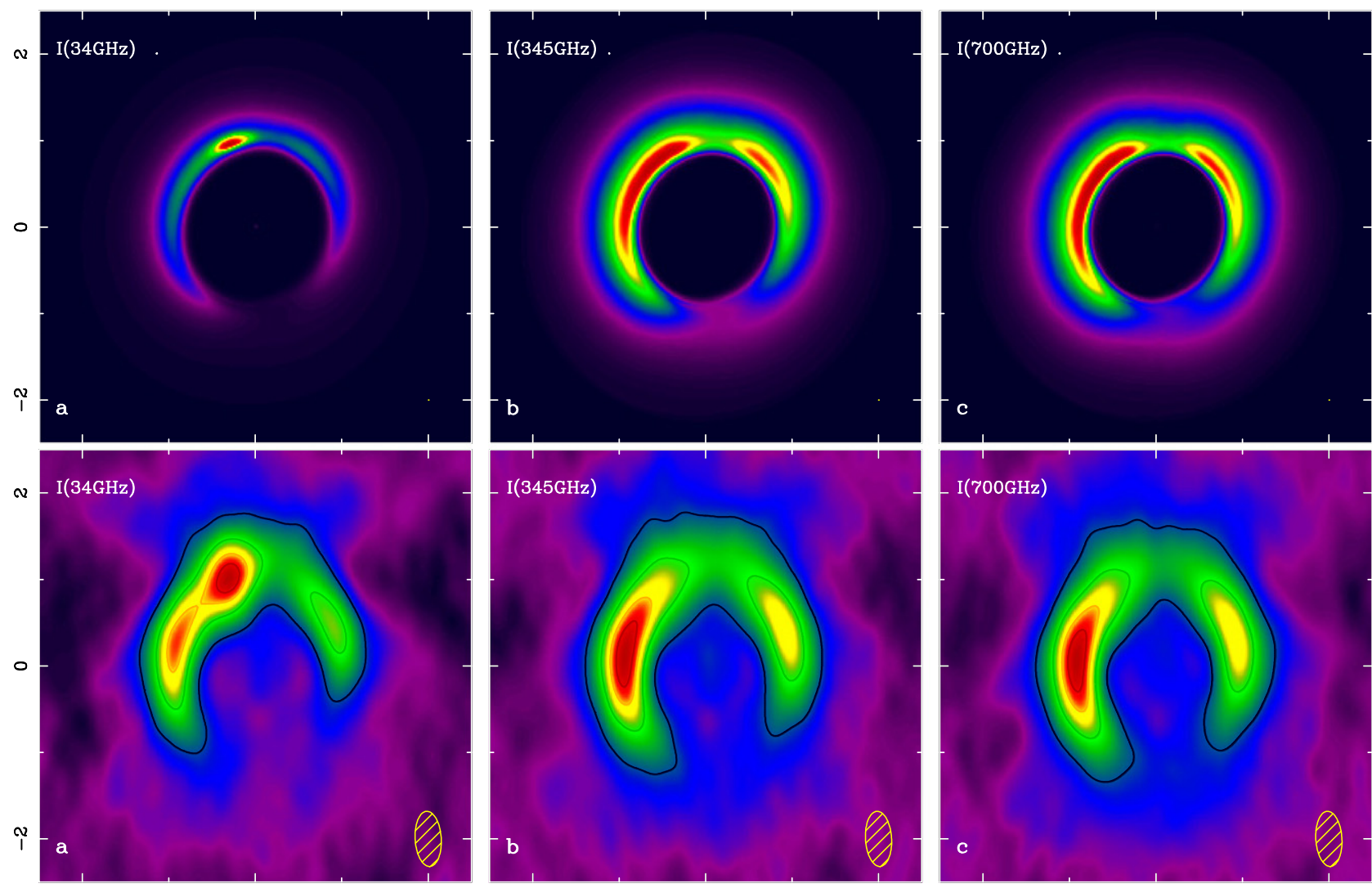

Figure 13. Predicted emergent continua at 34, 345, and $700 \mathrm{GHz}$. Upper row: raw RADMC3D prediction. Bottom row: predictions filtered by ATCA+uvmem response, with restoration in natural weights. Note the $11.5 \mathrm{hr}$ clump where the maximum grain sizes $(0.14 \mathrm{~cm})$ are found and which stands out at ATCA frequencies, but is absent at ALMA frequencies.

of $4.0 \times 10^{-4} M_{\odot}$ in amorphous carbon grains and of $1.6 \times$ $10^{-3} M_{\odot}$ in astrosilicate grains. To simulate the dust trap effect on the largest grains, we used the azimuthal trapping prescriptions from Birnstiel et al. (2013) on the dust grains larger than a critical size consistent with Equation (11) from Pinilla et al. (2012). We first define an axisymmetric gas distribution with a rounded disk wall, as previously described in Marino et al. (2015), but with adjusted parameters $\gamma=5$, $w=0.16$, and $r_{\mathrm{c}}=165.0 \mathrm{AU}$. We set a critical grain size $a_{\mathrm{c}}=0.14 \mathrm{~cm}$ for which bigger particles would be radially trapped in the pressure maximum, while smaller particles would follow the gas density background. This corresponds to the critical size at $140 \mathrm{AU}$ using Equation (11) from Pinilla et al. (2012). With a total gas mass of $0.18 M_{\odot}$ and an $\alpha_{t}$ viscous parameter of $3.0 \times 10^{-3}$, the Stokes number for $0.15 \mathrm{~cm}$ grains (our maximum grain size), at peak gas densities of $6.5 \times 10^{10} \mathrm{~cm}^{-3}$ and at $20 \mathrm{~K}$, is $\sim 0.002$ and so comparable to $\alpha_{t}$.

The above radial distribution is modulated in azimuth, using previously described formulae and symbols (Birnstiel et al. 2013; here we use $R_{s}=148 \mathrm{AU}$, and an azimuthal contrast in surface density of $c=20.0$ ). The scale height $H$ is given by

$$
H(r)=8.0\left(\frac{r}{130 \mathrm{AU}}\right)^{1.17}
$$

For the gas volume density, we used the standard vertical Gaussian distribution. The small grains, amorphous carbon and silicate grains smaller than $a_{\mathrm{c}}$, follow the same density field as the gas. The azimuthal distribution of big grains is calculated according to their Stokes number, a parameter to account for the gas turbulence $\alpha_{t}$ and the gas background (Birnstiel et al. 2013). For their radial distribution, we use a Gaussian parameterization to account for the spread caused by turbulence:

$$
\rho_{d, a}(r, \phi, z=0)=\rho_{d, a}(\phi) \exp \left[-\frac{\left(r-R_{s}\right)^{2}}{2 \sigma^{2}}\right],
$$

with $\sigma(a)=H_{r} \sqrt{\frac{\alpha_{t}}{S t(a)}}$. In order to compute the density as a function of height $z$, we use the same scale height as the gas. We investigated the implementation of grain settling, following a parameterization for the dust scale height (Armitage 2010). However, we found that in order to reproduce the projection effect at $\sim 1 \mathrm{hr}$, settling had to be very inefficient. Finally, the distribution of big grains is normalized to the global grain size distribution and total dust mass in big grains.

We used RADMC3D (Dullemond et al. 2015) to transfer the stellar radiation through the synthetic disk and predict emergent intensity maps, under the assumption of passive heating. We assumed a standard power-law distribution in grain sizes, with exponent -3.5 . The outer disk is composed of three different dust species representing a range in sizes described by $a_{\min }$ and $a_{\max }$.

1. $4.0 \times 10^{-4} M_{\odot}$ of amorphous carbon grains with radii $1<a<10 \mu \mathrm{m}$ with optical constants taken from $\mathrm{Li} \&$ Greenberg (1997). 

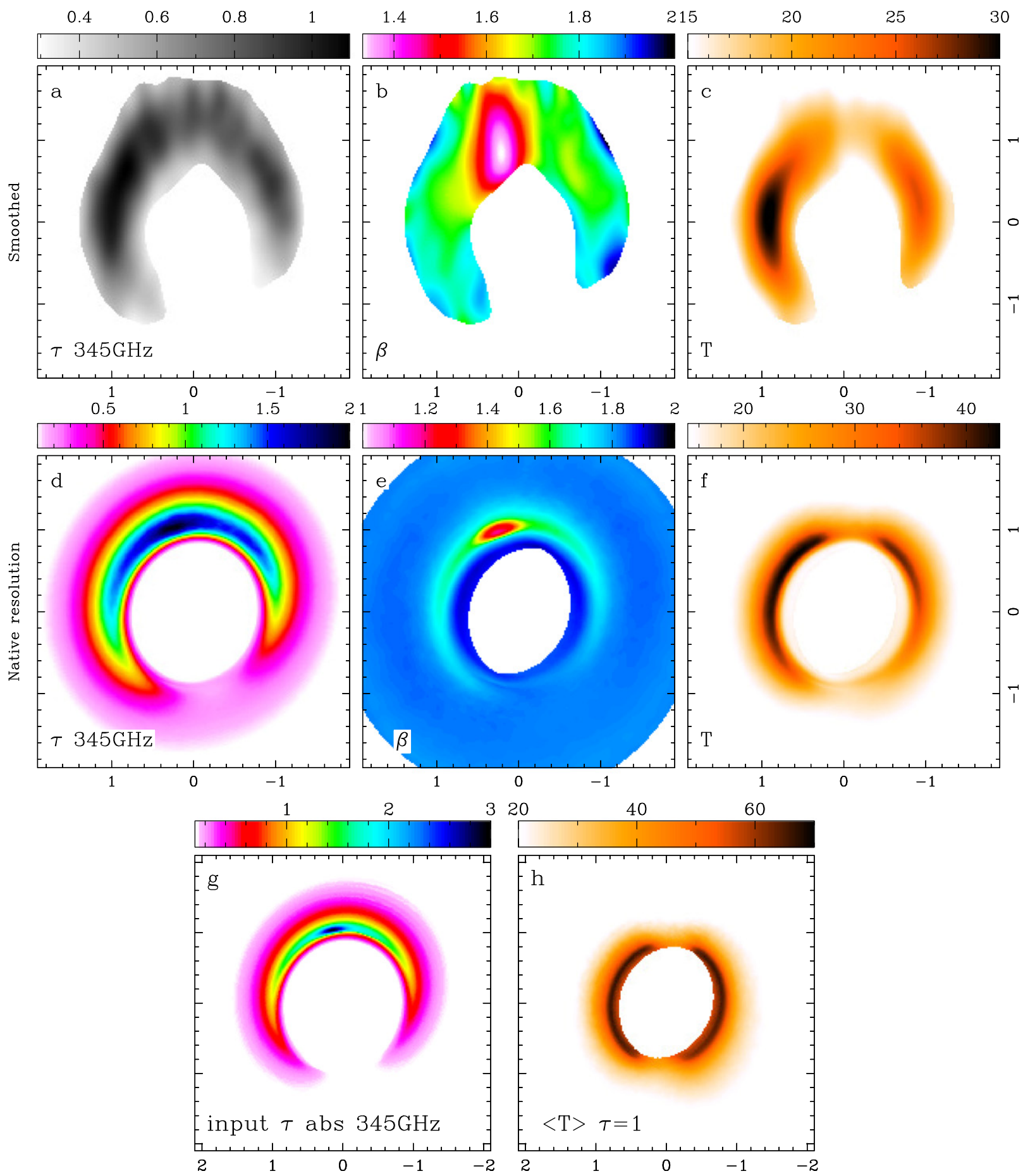

Figure 14. Graybody line-of-sight diagnostics calculated on the radiative transfer predictions, and comparison with input optical depth and temperatures. (a)-(c) Graybody diagnostics calculated after filtering by the ATCA+uvmem response. A mask has been applied to compute line-of-sight diagnostics at a fraction of $1 / 5$ times peak intensity. Panel (a) shows the optical depth map at the reference frequency of $345 \mathrm{GHz}$, (b) shows the line-of-sight emissivity index map $\beta_{s}(\boldsymbol{x})$, and (c) shows the line-of-sight temperature, $T_{s}(\boldsymbol{x})$. (d)-(f) Same quantities, as calculated on the radiative transfer predictions. (g), (h) Optical depth and average temperature maps used as input for the ray tracing. See text for the definition of average temperature.

2. $1.5 \times 10^{-3} M_{\odot}$ in "astronomical silicate" grains with radii of $100 \mu \mathrm{m}<a<a_{\mathrm{c}}$ and optical constants from Draine (2003).

3. $5.6 \times 10^{-5} M_{\odot}$ in "astronomical silicate" grains with radii of $a_{\mathrm{c}}<a<0.15 \mathrm{~cm}$ and optical constants from Draine (2003).
As summarized in Figure 11, the predicted integrated flux densities fall close to the observed dereddened SED extracted from previous SED fitting experiments (Verhoeff et al. 2011; Perez et al. 2015) and from the Herschel archive. Figure 12 shows that the model also reproduces the $H$-band scattered light images (this is an updated version of Figure 2 in Marino 
et al. 2015). We also included scattering for the purpose of calculating images at submillimeter frequencies and lower, but in the Henyey-Greenstein approximation (note, however, that the scattering cross sections calculated using Mie theory are probably overestimated in the submillimeter, given that the actual grains are probably irregular in shape, or even porous, and so far from the Mie spheres). The emergent intensity maps in Figure 13 show that the $34 \mathrm{GHz}$ emission exhibits a compact clump at the location of the dust trap and a more extended crescent that follows the submillimeter morphology. In order to simulate the ATCA response, we sampled the $u v$ coverage of the actual ATCA observations, but on the radiative transfer predictions, and we then averaged 100 uvmem reconstructions with different realizations of noise. This exercise produced sky maps that are remarkably similar to the ATCA-filtered observations. We also note that any flux loss is negligible.

\section{APPENDIX F \\ UNIFORM-SLAB DIAGNOSTICS ON THE SYNTHETIC DUST TRAP}

We also applied the line-of-sight diagnostic based on graybody models to the synthetic emergent intensities. As summarized in Figure 14, the basic features of the graybody diagnostics approximate those seen in the data. There is a mild extension of the optical depth map into the northern ansa, which could be emphasized with higher optical depths. The center of the dust trap coincides with a minimum in $\beta_{S}$. With this synthetic dust trap, we can also test for biases in the graybody diagnostics. The input optical depth map,

$$
\begin{aligned}
\tau(345 \mathrm{GHz})= & \int_{0}^{\infty} d s \rho_{g}(s) \\
& \int d a \sum_{i} n_{i}(a, s) \kappa_{i}(a, 345 \mathrm{GHz}),
\end{aligned}
$$

is remarkably consistent with the graybody values-at native resolutions. We can also compare the graybody temperature diagnostic, $T_{s}(\boldsymbol{x})$, with the line-of-sight average temperature as reported by RADMC3D after the thermal solution has converged:

$$
\begin{aligned}
\langle T\rangle= & \int_{0}^{\infty} d s \rho_{g}(s) \\
& \int d a \sum_{i} T(a, s) n_{i}(a, s) \frac{\kappa_{i}(a, 345 \mathrm{GHz})}{\tau(345 \mathrm{GHz}} .
\end{aligned}
$$

Figure 14 shows that the two temperature diagnostics are very nearly equal (to within 10\%). Limiting the integral to the depth $s$ corresponding to $\tau(345 \mathrm{GHz})=1$ resulted in essentially identical maps.

\section{REFERENCES}

Aikawa, Y., \& Nomura, H. 2006, ApJ, 642, 1152

Armitage, P. J. 2010, Astrophysics of Planet Formation (Cambridge: Cambridge Univ. Press)

Avenhaus, H., Quanz, S. P., Schmid, H. M., et al. 2014, ApJ, 781, 87

Ayliffe, B. A., Laibe, G., Price, D. J., \& Bate, M. R. 2012, MNRAS, 423,1450

Biller, B., Lacour, S., Juhász, A., et al. 2012, ApJL, 753, L38

Birnstiel, T., Dullemond, C. P., \& Pinilla, P. 2013, A\&A, 550, L8

Bruderer, S., van der Marel, N., van Dishoeck, E. F., \& van Kempen, T. A. 2014, A\&A, 562, A26

Canovas, H., Ménard, F., Hales, A., et al. 2013, A\&A, 556, A123

Casassus, S., Cabrera, G. F., Förster, F., et al. 2006, ApJ, 639, 951

Casassus, S., Perez, M. S., Jordán, A., et al. 2012, ApJL, 754, L31

Casassus, S., van der Plas, G., Perez, S. M., et al. 2013, Natur, 493, 191

Christiaens, V., Casassus, S., Perez, S., van der Plas, G., \& Ménard, F. 2014, ApJL, 785, L12

Close, L. M., Follette, K. B., Males, J. R., et al. 2014, ApJL, 781, L30

Draine, B. T. 2003, ApJ, 598, 1026

Dullemond, C., Juhasz, A., Pohl, A., et al. 2015, RADMC3D v0.39 http:// www.ita.uni-heidelberg.de/dullemond/software/radmc-3d/

Fouchet, L., Gonzalez, J.-F., \& Maddison, S. T. 2010, A\&A, 518, A16

Fujiwara, H., Honda, M., Kataza, H., et al. 2006, ApJL, 644, L133

Fukagawa, M., Tamura, M., Itoh, Y., et al. 2006, ApJL, 636, L153

Fukagawa, M., Tsukagoshi, T., Momose, M., et al. 2013, PASJ, 65, L14

Kuiper, G. P. 1951, PNAS, 37, 1

Li, A., \& Greenberg, J. M. 1997, A\&A, 323, 566

Lyra, W., Johansen, A., Zsom, A., Klahr, H., \& Piskunov, N. 2009, A\&A, 497,869

Lyra, W., \& Lin, M.-K. 2013, ApJ, 775, 17

Marino, S., Perez, S., \& Casassus, S. 2015, ApJL, 798, L44

McMullin, J. P., Waters, B., Schiebel, D., Young, W., \& Golap, K. 2007, in ASP Conf. Ser. 376, Astronomical Data Analysis Software and System XVI, ed. R. A. Shaw, F. Hill, D. J. Bell (San Francisco, CA: ASP), 127

Mittal, T., \& Chiang, E. 2015, ApJL, 798, L25

Ohashi, N. 2008, Ap\&AA, 313, 101

Paardekooper, S.-J., \& Mellema, G. 2006, A\&A, 453, 1129

Pérez, L. M., Isella, A., Carpenter, J. M., \& Chandler, C. J. 2014, ApJL, 783, L13

Perez, S., Casassus, S., Ménard, F., et al. 2015, ApJ, 798, 85

Pinilla, P., Birnstiel, T., Ricci, L., et al. 2012, A\&A, 538, A114

Pollack, J. B., Hubickyj, O., Bodenheimer, P., et al. 1996, Icar, 124, 62

Rameau, J., Chauvin, G., Lagrange, A.-M., et al. 2012, A\&A, 546, A24

Regály, Z., Juhász, A., Sándor, Z., \& Dullemond, C. P. 2012, MNRAS, 419, 1701

Rodigas, T. J., Follette, K. B., Weinberger, A., Close, L., \& Hines, D. C. 2014 ApJL, 791, L37

Sándor, Z., Lyra, W., \& Dullemond, C. P. 2011, ApJL, 728, L9

Sault, R. J., Teuben, P. J., \& Wright, M. C. H. 1995, in ASP Conf. Ser. 77, Astronomical Data Analysis Software and Systems IV, ed. R. A. Shaw, H. E. Payne \& J. J. E. Hayes (San Francisco, CA: ASP), 433

van der Marel, N., van Dishoeck, E. F., Bruderer, S., et al. 2013, Sci, 340, 1199

van der Plas, G., Casassus, S., Ménard, F., et al. 2014, ApJL, 792, L25

van der Wiel, M. H. D., Naylor, D. A., Kamp, I., et al. 2014, MNRAS, 444, 3911

Verhoeff, A. P., Min, M., Pantin, E., et al. 2011, A\&A, 528, A91

Weidenschilling, S. J. 1977, MNRAS, 180, 57

Wilson, W. E., Ferris, R. H., Axtens, P., et al. 2011, MNRAS, 416, 832

Zhu, Z., \& Stone, J. M. 2014, ApJ, 795, 53

Zuckerman, B., Forveille, T., \& Kastner, J. H. 1995, Natur, 373, 494 